\title{
Complex Dynamic Scene Perception: Effects of Attentional Set on Perceiving Single and Multiple Event Types
}

\author{
Thomas Sanocki and Noah Sulman \\ University of South Florida
}

\begin{abstract}
Three experiments measured the efficiency of monitoring complex scenes composed of changing objects, or events. All events lasted about $4 \mathrm{~s}$, but in a given block of trials, could be of a single type (single task) or of multiple types (multitask, with a total of four event types). Overall accuracy of detecting target events amid distractors was higher for single event types relative to multiple types. Multiple event types were processed reasonably well when each event type was restricted to its own region, and much worse when event types were mixed in location. In most task conditions, observers reached an optimal level of performance (optimal attentional set). After one target was identified, performance for other targets dropped markedly and then recovered to optimal levels. However, set was not optimized when task locations were intermixed. The results support the idea that attentional set determines the efficiency of event perception in complex scenes. Although single event set was most efficient, there can be a reasonably efficient set for multiple event types.
\end{abstract}

Keywords: scene perception, event perception, attentional set, multitasking, attention

How does the efficiency of perceiving objects in scenes vary with the number of observer goals, or attentional sets? The present experiments addressed this question with complex, dynamic scenes and objects-animated displays composed of many simple changing objects, or events. Set was manipulated by presenting either a single event type or multiple event types. Observers searched for target events amid distractor events, and overall perceptual efficiency was indexed by the hit rate for target events. An attentional set hypothesis predicts that perceptual efficiency should be higher when observers can be set for a single event type than when observers must be set for multiple event types, requiring multiple visual sets. This research was motivated by several literatures, including those on top-down processing and attention, and scene perception.

\section{Conceptual Background}

\section{Top-Down Processing}

The topics of everyday scene perception and top-down influences have been central to cognitive approaches to vision (e.g., Broadbent, 1977; Bruner, 1957; McClelland \& Rumelhart, 1981; Neisser, 1967; Schyns, Goldstone, \& Thibaut, 1998). Everyday scene perception is important ecologically because it is something people often do (see Neisser, 1976). Top-down effects are significant because they are cognitive mechanisms that can have strong

This article was published Online First November 26, 2012.

Thomas Sanocki and Noah Sulman, Department of Psychology, University of South Florida.

Correspondence concerning this article should be addressed to Thomas Sanocki, Psychology PCD 4118, University of South Florida, 4202 E. Fowler Avenue, Tampa, FL 33620. E-mail: sanocki@usf.edu influences on perceptual processing. However, the relative strength of top-down processing remains to be determined, because research has not adequately explored potentially relevant factors such as scene complexity, process preparation, and event time scale. Combining these considerations motivates an experimental paradigm that can be termed complex, dynamic scene perception.

\section{Attentional Set Approach to Scene Perception}

The core idea in this top-down approach is that everyday perception is an active process whose success depends on the state (set) of the perceiver (e.g., Most, Scholl, Clifford, \& Simons, 2005; Postman \& Bruner, 1949). State is a deep concept involving multiple factors, including expertise (e.g., Goldstone, Braithwaite, \& Byrge, 2012) and motivation and beliefs (e.g., Balcetis \& Dunning, 2006). When these factors are constant, the preparation of visual processing components can have a strong influence on perception (e.g., Postman \& Bruner, 1949; Walther \& Li, 2007). We manipulated the number of event types to which observers must attend. When there is a single event type with one target stimulus, the observer can prepare for the stimulus in an optimal manner. This increases the likelihood that observers will become conscious of instances of the target, relative to unexpected or unattended stimuli (e.g., Mack, 2003; Most et al., 2005; Simons \& Chabris, 1999). In this context, attentional set can be thought of as control settings describing target visual features or properties (e.g., Folk, Remington, \& Wright, 1994; Leber, Kawahara, \& Gabari, 2009; Most et al., 2005). Single-event conditions should require a single description for the one event type, whereas multievent conditions should require multiple descriptions, or one more general description. Perceptual efficiency should be reduced in multievent conditions because of the more general descriptions used.

Attentional set is important in scene perception because of the nature of scenes and scene perception. We argue that scenes and 
scene perception defy narrow definition; at their most essential levels, they are characterized by their ranges - by the variation between scene types and between observer behaviors (cf. Henderson \& Hollingworth, 2003; Greene \& Oliva, 2009; Torralba, Oliva, Castelhano, \& Henderson, 2009; Tsotsos, 2001). Scenes differ greatly on spatial factors such as scale and complexity, and on temporal factors as well. Observer activities may differ even more.

Given the wide range of scenes and observer activities, the mechanisms of scene perception are likely to be complex and powerful while also being adaptable. If so, change in mechanisms due to scene or task should be nontrivial. This is a fundamental implication of the attentional set hypothesis (e.g., Most et al., 2005; Neisser, 1976). And it corresponds to the well-studied cost of task switching-reduced performance caused by the reconfiguration of processes when task changes (e.g., Monsell, 2003). Costs of task switching have been examined in detail within a large literature using mostly simple stimulus situations (e.g., Monsell, 2003; Van Loy, Liefooghe, \& Vandierendonck, 2010). Current work on task switching is focusing on the specific processes that underlie these costs, and a number of hypotheses remain viable (e.g., Kiesel et al., 2010; Van Loy et al., 2010; Vandierendonck, Liefooghe, \& Verbruggen, 2010).

Large effects of attentional set have also been found with complex stimuli, including real-world scenes and events, such as the appearance of a gorilla (e.g., Most et al., 2005; Simons \& Chabris, 1999; Walther \& Li, 2007). Typically in these studies, there is a critical stimulus (e.g., gorilla) that can be perceived when observers expect it or have adopted a general set. However, in the main conditions, observers are induced to be set for another complex task; the gorilla is unexpected and requires a change of visual task. In these conditions, the gorilla is often not noticed or consciously perceived (e.g., Macdonald \& Lavie, 2008; Most et al., 2005; Simons \& Chabris, 1979; White \& Davies, 2008; see also Mack \& Rock, 1998). In fact, unexpected stimuli may not cause attentional capture (e.g., Folk, Remington, \& Wright, 1994).

Although lack of noticing is consistent with the attentional-set hypothesis, the effects are somewhat limited in nature. Because the critical events are unexpected, they occur only once in most experiments. ${ }^{1}$ Also, the responses are often indirect (e.g., answers to post hoc questions), and can be influenced by expectation or memory. In the present experiments, we sought to extend attentional-set effects to frequent, expected stimuli. The goal was to measure repeated missings of expected gorillas.

\section{The Importance of Stimulus and Task Complexity}

Stimulus complexity has major effects on perceptual processing, ${ }^{2}$ and top-down processing becomes increasingly important as the stimulus situation gets more complex. For example, scene categorization becomes slower and more difficult when varying foreground objects are present (Walker, Stafford, \& Davis, 2008). From a computational viewpoint, the challenge of computing interpretations from scenes in spite of their complexity is a significant, perhaps defining feature of general scene perception (Tsotsos, 1990, 2001).

Perceptual processing also varies in complexity. Much sceneperception research has focused on processes such as scene categorization, which are convergent in nature-a stimulus image is processed to arrive at a single consensus label—which denotes, for instance, the category, or the presence of an object in the scene. Such paradigms do not capture some major complexities of everyday scene perception. First, objects in live scenes change over time, creating events. Events can have many complexities, including time scale and history (see next section). Second, convergent paradigms require a single interpretation within a single task. In contrast, natural scenes vary in how many interpretations they support and how easy the interpretations are. Natural observers may engage in a variety of perceptual tasks within a period of time. These factors greatly compound the complexity of everyday scene perception, and are largely unexplored in scene-perception research. ${ }^{3}$

Costs of changing perceptual task may be more likely to appear in complex situations. Our scenes were composed of dynamic objects and involved divergent visual tasks. However, we avoided stimulus or response ambiguity because we did not want switching costs to be influenced by uncertainty, which can inflate response times to particular features, objects, and experimental situations (e.g., Sanocki \& Oden, 1991).

\section{Events and Their Time Scales}

Most of the stimulus scenes in scene-perception research consist of objects, surfaces, and their layouts. However, natural scenes are live, and objects within them often change over time, producing events. Events compound the complexity of scene perception, by adding temporal dimensions of variation. There is a growing literature on event perception, and it involves a wide variety of event types, at a variety of temporal scales (e.g., Zacks \& Tversky, 2001). The temporal structure of events plays a key role in the organization of mental life, and expectations regarding the temporal structure and scale of perceptual events are available in early in life (Baillargeon, 2004; Hespos \& Baillargeon, 2001; Tversky, Zacks, \& Hard, 2008). Temporal scale, like display complexity, may be a critical variable for determining the nature of perceptual processing.

In the present case, we decided to begin by examining a single time scale that, on intuitive grounds, seems to be near optimum for human scene perception-the time scale of several seconds. As noted, many human events take place over seconds, and people like to watch events that last seconds. Our events were simple animated objects; research indicates that animated objects can be life-like and meaningful (e.g., Michotte, 1950/1991, Gao, Newman, \& Scholl, 2009). The events were designed to be simple, predictable, and of a similar time scale, so that set effects would not depend on uncertainty about event structure or differences in times scales between events.

\footnotetext{
${ }^{1}$ Misperception has been studied for events with frequencies over one, but the events are peripherally presented (e.g., Macdonald \& Lavie, 2008) or the measure is indirect (Folk et al., 1994).

${ }^{2}$ For example, effects of display size (e.g., Schneider \& Shiffrin, 1977), perceptual load and dilution (e.g., Lavie, Hirst, \& de Fockert, \& Viding, 2004; Tsal \& Benoni, 2010), and crowding.

${ }^{3}$ Other significant complexities of scene perception include the challenges of segmenting objects and events from background information, and the challenges of coordinating the different scene views obtained by an active observer across eye movements and changes of position.
} 


\section{Surveillance as an Intense Scene-Perception Process}

One additional motivation concerns scene surveillance. Security is critically important for communities, both in present times and throughout most of human history. Modern surveillance methods present large amounts of information to observers, on multiple screens, or display cells. The widespread use of multiple displays suggests that this is an effective way of presenting scene information, although there are limits (e.g., Sulman, Sanocki, Goldgof, \& Kasturi, 2012). In the present experiments, we presented the events on a grid on four display cells (see Figure 1). Information about performance can be useful for the practical problem of providing security, as well as for perceptual theory.

\section{Summary}

Complex, dynamic scenes are especially interesting because their rich, many-dimensional structures seem to invite top-down influences. With this belief in mind, we developed the following experiments.

\section{Introduction to the Experiments}

The empirical goal was to measure overall perceptual efficiency as a function of the number of event types. In single-task conditions, a single event type occurred in each of the four display cells, whereas there were four event types in the multitasking condition (see Figure 1). Each trial involved a 60-s stream during which 144 events occurred asynchronously. The event types required the same decision and response rules while varying markedly on visual factors. The events had different visual properties to minimize confusion between tasks. In the task-switching literature, each event type would be termed univalent, because the event tokens had only one meaning. Two event types were termed ventral events because their token instances remained stationary

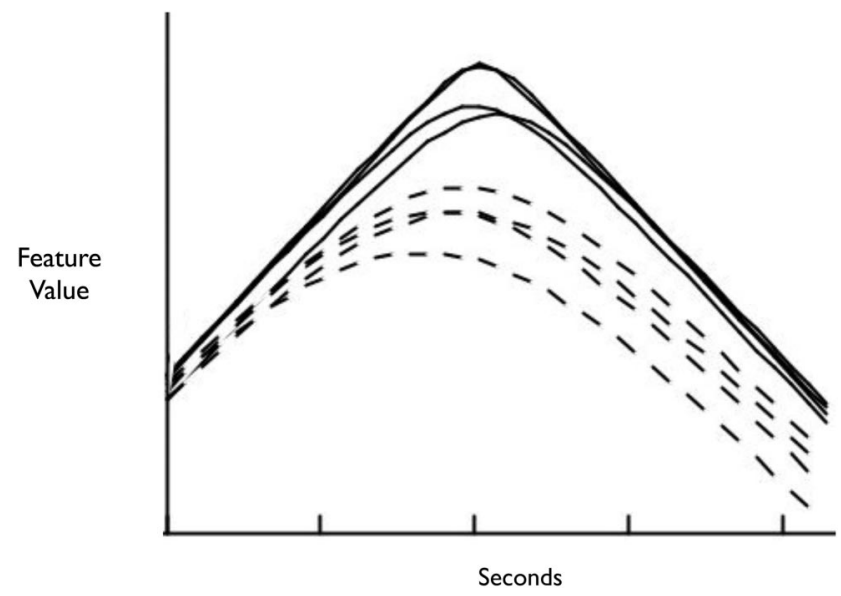

Figure 2. Schematic event trajectories for targets (solid lines) and distracters (dashed lines).

and the critical dimension was a ventral property (color and shape). The other two event types were termed dorsal events because their tokens moved and the critical dimension was location or type of motion. Tokens for each task had a unique color and shape.

\section{Event Lifetimes}

Each object token started its lifetime as a distractor and then proceeded to change (increase and then decrease) along the single, critical feature dimension, during a 4-s lifetime. If the increase was large enough, the token became a target for a period of time. Figure 2 shows schematic event trajectories-lifetimes for targets (solid lines) and distractors (dashed lines). Observers responded to targets with a key press, as they ignored distractors. For example,
A) Single-task condition (location task)

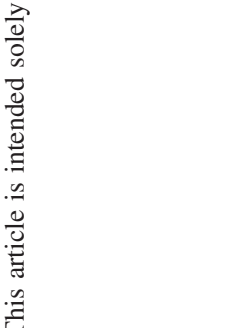

B) Multi-task condition (motion, color, location, shape)

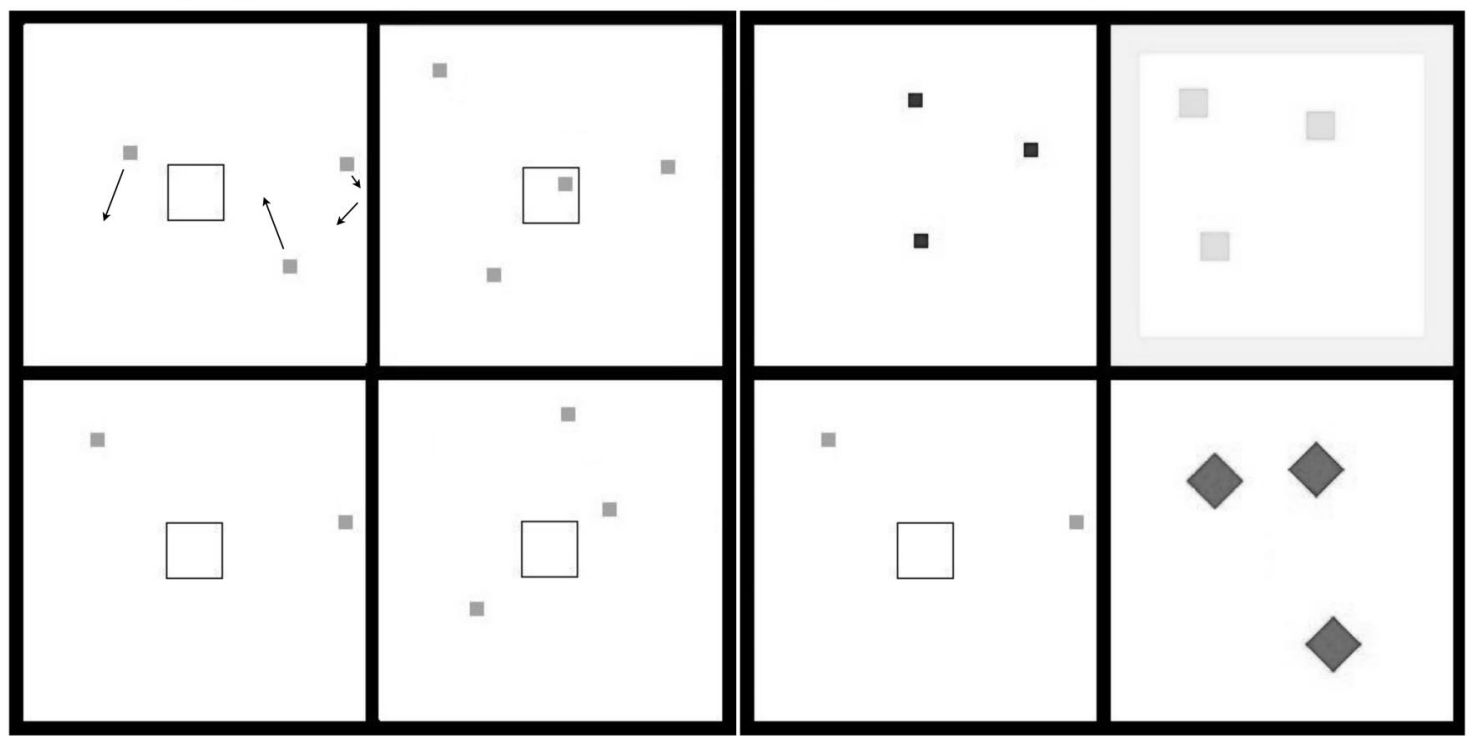

Figure 1. Main conditions Experiment 1. A) Single-task condition, with possible pathways shown for one cell. B) Multi-task condition, with tasks labeled above (clockwise from upper left). 
in the dorsal location task, the tokens moved in linear pathways (usually oblique) up or down the display cell (see Figure 1). The critical feature was location-a token became a target if it entered a central box. The target-distractor distinction was obvious when observers attended to the tokens.

Although each event had a predictable trajectory, observers had to fixate near a token to process it optimally. This meant that observers had to move their eyes and attention from cell to cell throughout a trial.

\section{Interference Between Targets Close in Time?}

An additional empirical issue was how the detection of one event might influence detection of other events occurring close in time. The timing of targets was distributed randomly throughout trials, producing intertarget intervals (ITIs) ranging from 0 to beyond $10 \mathrm{sec}$. Temporally close targets may interfere with each other, perhaps in a manner roughly analogous to the attentional blink, which is obtained with very rapid stimulus presentations (Chun \& Potter, 1995; Raymond, Shapiro, \& Arnell, 1992). Our goal was to collect basic data on intertarget relations, which may be useful for understanding attentional set.

\section{General Method}

Observers participated in individual sessions that began with training on each of the four event types. Then single-task blocks were run, followed by multitask blocks, and then a repeat of the single-task blocks, to permit assessment of learning across the test blocks.

\section{Stimulus Displays}

The stimulus for each trial was a 60-s stream composed of 4-s events, or event tokens. The 144 tokens on a trial were divided among four adjacent display cells (see Figure 1). Eight of the 144 tokens turned into targets. Observers were instructed to respond to targets only by pressing a key. In Experiments 1 and 2, the key location corresponded to display-cell location. There were four token types, one token type for each task. The display was $14 \times 14$ $\mathrm{cm}$, with a visual angle of $29.5^{\circ}$ at the viewing distance of (approximately) $54 \mathrm{~cm}$. Figure 1 reproduces example displays in accurate relative scale, but without color. An iMac computer with standard keypad was used; the experiment was programmed in RealBasic, 2008 (Austin, TX).

A token's lifetime began when it appeared and then lasted about $4 \mathrm{sec}$, when it disappeared. During most of a given trial, there were 12 tokens in the display, at varying stages of their lifetimes. When a given token reached the end of its lifetime, it disappeared and then a new token appeared in the same display cell, $1 \mathrm{~s}$ later. Thus, consecutive tokens within a cell formed token streams, and the stream spanned the 60-s trial. Targets were constrained to appear only in lifetimes $2-11$ of a token stream; the first and last lifetimes were buffer periods with no targets. Asynchrony was produced by delaying the start of token streams (the appearance of the first token) relative to each other.

Location task. In this task, gray square tokens traveled upward or downward at the same rate, in a linear path at varying angles (see Figure 1a). Proximity to a central critical box was the critical dimension; targets traveled through the box and distractors missed the box. The possible trajectories were predefined offline; a token's trajectory was chosen from those produced by crossing 55 evenly spaced starting locations at the top or bottom of display cells with 30 motion angles (between $-45^{\circ}$ and $+45^{\circ}$, relative to upright). When a token hit the wall, it was deflected with a reflection of its path. The 1650 possible trajectories were sorted into those that directed tokens through the central target region (target events) and those that did not (distractor events). Close cases, where tokens grazed the central region by $.25^{\circ}$ or less, were removed. This left 299 target paths and 1106 distractor trajectories. Token pathways were randomly selected from these (with no replacement within trials).

Motion task. Square blue tokens moved across the screen left to right, with varying degrees of vertical wobble (perturbation up or down; see Figure 1b, upper left). Targets had more extreme wobble, appearing "drunk." The horizontal screen dimension was divided into eight sections (octants). For each token, at every new octant the vertical motion component reversed sign (up vs. down) and the slope changed. The vertical slope increased with each octant through the sixth, and then decreased for the last two. The slopes were greater for targets than for distractors, producing the appearance of a stronger wobble, especially in octants 5 and 6 .

Color task. Square color tokens appeared and remained at random locations; their colors changed through color space across the lifetime, beginning as green-yellow and then changing toward pure yellow and then back to green-yellow. Targets changed more toward yellow, to match the yellow border of the color-task display cells (see Figure 1b, upper right). The color changes consisted of 40 gradual steps, beginning at a red-green-blue value of 127, 255, 0 , on the red-green-blue (RGB) scale $(0=$ black, $255=$ white $)$. The red component was increased for the first 20 steps of a token lifetime and then decreased for the last 20 steps. Distractors and targets differed in the range of change in the red value; distractors changed in value between 3 and 6 at each step, whereas targets changed between 6 and 8.5; only targets reached the defining value of $(255,255,0)$, which matched the border. Targets varied somewhat in how long they remained at the defining value.

Shape task. Red diamond-shaped tokens appeared and remained at random locations; their shapes changed in concavity across the lifetime. Tokens began as fat diamonds (slightly convex sides) and changed to become star-like (very concave sides), with targets defined by increased concavity. Concavity decreased for the first 20 steps of a lifetime and increased for the last 20. The shape pathways were chosen from 20 distractor and 20 target pathways generated before the experiment with varying sizes of shape steps. As in the other tasks, only targets came close to and crossed the maximum value.

\section{Main Test Procedure}

There were four different single-task conditions, one for each task/event type. At the start of a trial, the 12 token streams for that trial were assigned to display cells, with each display cell being randomly assigned a density of streams of 2, 3, 3, or 4 (for a total of 12). For new tokens, entry points and locations were random within ranges inside the cell. The eight target tokens on a trial were randomly assigned to token streams and lifetimes within streams (excluding the two buffer periods). This method distributed targets 
evenly throughout both space and time. Overlap between target lifetimes, which could produce competition between targets, ranged between 0 (same peak of critical feature function) to beyond $10 \mathrm{sec}$. The multitask conditions used the same constraints as the single-task conditions except that the 12 token streams were divided among the four event types $(2,3,3$, or 4 per event, for a total of 12, randomly assigned at the beginning of each trial). For a given observer, each event type was assigned to a display cell that remained constant throughout the entire multitasking phase (grouped multitasking in Experiments 1 and 3).

Observers were instructed to press the spacebar to initiate a trial. They were then free to respond throughout the trial, by pressing the appropriate key (see below). A trial ended as the last token lifetime ended. There was no feedback during this main test phase. Observers learned about the task and target frequencies during the initial training period. During testing, responses were interpreted as hits if they indicated the correct cell (Experiments 1 and 2) and if they occurred within a 4-s window centered on the peak of the target's critical feature variation. We used this window to allow anticipatory responses, because event trajectories were predictable. In general, a target token's critical property first diverged from the distractors at about $1 \mathrm{~s}$ into its lifetime (e.g., Figure 2). The peak was reached between 2 and $3 \mathrm{~s}$ into the lifetime. The 4-s response window allowed anticipatory responses and continued out to about $3 \mathrm{~s}$ after the first divergence of the critical property. If a second response occurred within the window but no new target had been presented, it was scored as a false alarm (FA).

\section{Training}

Sessions began with a training phase for each task. In training, observers monitored a single display cell for 60-s trials, with 2-4 token streams (randomly determined) and eight targets in the cell. Responses were scored as during the main test phase. However, for each task, auditory feedback was provided during the first four trials (an incorrect or correct tone for each response). Then feedback was withheld for two more trials during which performance was measured. Performance was high for most observers (hit rate above $95 \%$, FA rate $<2 \%$ ). Observers who did not perform well on each task during this nonfeedback phase were removed from analyses; the criterion for inclusion was a hit rate above $75 \%$ and FA rate below $10 \%$

\section{Order of Conditions}

The order of the four tasks during training and the single-task conditions was determined randomly for each observer and main- tained throughout the entire session. The test conditions followed the complete training phase. There was a single-task condition with each event type, followed by the multitask condition, and then a repeat of the single-task conditions. This design (summarized in Table 1) allowed us to measure overall changes in performance during the session by comparing the initial and late single-task levels ( $\mathrm{a}$ and $\mathrm{c}$ in Table 1), and also provided a balanced contrast of single- and multitask performance ( $b$ vs. $a+c)$. In Experiments 1 and 2, the first single-task phase consisted of (a) for each event type, four practice trials followed by four test trials. The multitask phase consisted of (b) four practice trials followed by 16 test trials. The second single-task phase consisted of (c) for each event type, one practice trial followed by four test trials.

\section{Experiment 1}

The purpose of Experiment 1 was to measure the possible cost of switching between event types during a trial. We contrasted performance with single event types (e.g., Figure 1A) with multitask performance, for four event types, with one event type in each cell (Figure 1B). In each condition, there were eight target tokens and 136 distractor tokens. In the multitask conditions, there were two target-tokens and an average of 34 distractor tokens for each task. If scene perception is most efficient when a single attentional set can be used throughout a trial, then performance should be highest in single-event (i.e., single-task conditions). When the attentional set must be continuously changed for multiple events (multiple-task condition), performance should be markedly reduced.

\section{Method}

Observers were instructed to respond only to targets, by pressing a key that corresponded spatially to the four cells (on the standard keypad; "4" for top left, "5" for top right, " 1 " for bottom left, " 2 " for bottom right). The response was a hit only if both cell and timing were correct (see General Method). Fifteen college students (nine women) participated in exchange for course credit, from the University of South Florida. The data for an additional two participants were not analyzed because they did not pass the training criteria.

\section{Results}

Hit rates provide a good measure of overall efficiency because FA rates were low in the test conditions $(<1 \%$ most conditions). Hit rates and sensitivity and bias values are reported in Table 2 .

Table 1

Session Timeline in Experiments 1 and 2

\begin{tabular}{llll}
\hline \multicolumn{4}{c}{ TIME $\rightarrow(\sim 60$ min total $)$} \\
\hline & \multicolumn{4}{c}{ Test phase (four display cells) } \\
\cline { 2 - 4 } Training (one cell) & \multicolumn{1}{c}{ (a) } & (b) & (c) \\
\hline Learn-test for task & Single task, 4-cell & Multitask, 4-cell & Single task, 4-cell \\
$1,2,3$, then 4 & $1,2,3$, then 4 & $1-4$ one per cell & $1,2,3$, then 4 \\
\hline
\end{tabular}

Note. Tasks are numbered in order (1-4); event types were randomly assigned to order for each observer. 
Table 2

Sensitivity Results in Main Conditions of Each Experiment

\begin{tabular}{lcccc}
\hline & Hits (\%) & FA's (\%) & $d$ ' & $C$ \\
\hline Experiment 1 Single-event & 78.4 & 0.8 & 3.53 & 0.87 \\
Multievent & 64.3 & 0.9 & 2.97 & 1.09 \\
Experiment 2 Single-event & 79.3 & 0.8 & 3.50 & 0.81 \\
Multievent & 45.2 & 2.0 & 2.17 & 1.21 \\
Experiment 3 Single-event & 85.5 & 0.3 & 4.07 & 0.81 \\
Grouped Multievent & 64.9 & 1.0 & 2.75 & 0.97 \\
Distributed Multievent & 53.1 & 1.0 & 2.48 & 1.17 \\
\hline
\end{tabular}

The main statistical analyses were planned comparisons between task conditions and factorial analysis of variance (ANOVA).

The hit rate was high at the end of training with one-cell displays and a single task (96.5\%). When stimulus complexity was increased during the test phase to four cells with a single task, performance was reduced to an average of $78.4 \%, F(1,14)=7.80$, $p<.001$. The reduction suggests that stimulus complexity strained the observers' resources in these single-task conditions.

When event types varied in the multitasking condition, performance was further decreased, to $64.3 \%$. This $14.1 \%$ cost was highly reliable: $S E$ of cost $=3.0 \% ; F(1,14)=22.0, p<.001$; $\eta_{\mathrm{p}}^{2}=.61$. More concretely, subjects detected an average of 6.3 of eight targets in the single-task conditions, and 1.2 fewer targets in the multitasking condition. Thus, there was a cost for switching between multiple event types in this experiment.

Consistency across event type. Table 3 shows hit rates broken down by event type (task). There was the main effect of task condition reported above, but no main effect of event type, $F(1$, $14)=1.69, p>10$. The interaction of event type and task condition was reliable, $F(3,42)=6.03, p<.01 ; \eta_{\mathrm{p}}^{2}=.30$. For three of the event types, performance was higher in the single-task conditions than in the multitask condition. The exception is the location event, where there was a small advantage in multitask conditions in this experiment. The lack of cost for the location event may reflect a confound in the location event-there was only one critical location in the multitasking condition (in the one cell for that event type), but four in the single-tasking conditions (one for each cell; see Figure 2b). Additional evidence on this issue is provided by the following experiments. Tentatively, although noting a confound for the location task, we conclude that the multitasking costs were generally consistent across tasks.

Effects of intertarget-interval (ITI). Do target events that occur close in time interfere with each other? We divided up the data by ITI, after first separating out the data for initial (first) targets on each trial. For subsequent targets, the temporal interval after the previous target ranged from 0 (two target events with peak critical features values at the same time) to $10 \mathrm{~s}$ and beyond.

Table 3

Hit Rates for Each Event Type and Task Condition in Experiment 1

\begin{tabular}{lcccr}
\hline & Color & Shape & Motion & Location \\
\hline Single-event & 0.78 & 0.85 & 0.79 & 0.73 \\
Multievent & 0.63 & 0.65 & 0.54 & 0.75 \\
Cost & 0.15 & 0.19 & 0.25 & -0.03 \\
\hline
\end{tabular}

The data up to $10 \mathrm{~s}$ were divided into one s ITI windows and are shown in Figure 3, labeled by the longest ITI (e.g., the first interval includes ITIs of $0-1 \mathrm{~s}$ ). Shorter ITIs contained more trials - the 1-s window contained $22 \% \%$ of the trials, and frequency gradually decreased to (e.g.) $6 \%$ at $6 \mathrm{~s}$ and $3 \%$ at $10 \mathrm{~s}$. The 10 ITIs shown captured $89 \%$ of the trials with noninitial targets.

If there was interference, we expected it to occur at the shorter ITIs. Performance may then return to asymptotic or baseline levels as ITI increased. There was in fact a decrement at the two shorter ITIs, as can be seen in Figure 3. In an ANOVA with task condition and ITI as factors, there was the main effect of task condition reported above, and a main effect of ITI, $F(9,135)=3.01, p<$ $.01 ; \eta_{\mathrm{p}}^{2}=.17$. The interaction of these variables was not reliable, $F(9,135)<1$. To measure the magnitude of decrements at short ITIs, we used as baseline the longer eight ITIs (ITIs 3-10 sec). At the shortest ITI, the decrement was $15.1 \%, t(15)=5.36, p<.001$. At the $2 \mathrm{~s}$ ITI, the decrement was $6.2 \%, t(15)=2.14, p=.049$. Thus, there was a decrement for temporally close targets. This decrement may be analogous to the attentional blink in research on temporal attention. However, the present decrement was much longer (over $2 \mathrm{~s}$ ) than in research with rapidly presented stimuli, where the effect typically ends after about $0.5 \mathrm{~s}$.

Learning effects during the session. How much did performance change during the test session? The single-task blocks were run before and after the multitask block (see Table 1), to bear on this issue. Performance stayed constant across the early and late single-task blocks, averaging $78.6 \%$ and $78.3 \%$, respectively $(t<$ 1). Thus, after observers learned the tasks during training, performance was generally constant across the experiment.

\section{Discussion}

The results indicate that there was a highly reliable cost of multievents-observers were $14 \%$ less efficient when monitoring four types of events than when monitoring single events. The results are consistent with the attentional-set hypothesis and the claim that switching between events would be costly. These costs appear to be perceptual rather than response-related because the decision and response rules were the same across task conditions.

The results also included large interference effects for targets occurring close together in time, in a manner roughly analogous to the attentional blink. However, the present deficit lasted over $2 \mathrm{~s}$. Once the deficit is over, there appears to be an asymptotic period from 3-10 s. This may represent an optimized set in that condition. We discuss these effects in more detail at the end of the experiments, because combining data across experiments provides a more systematic body of evidence.

Although the $14 \%$ multitasking cost was reliable and reasonable in size, we suggest that the magnitude is not as large as one might expect if a single attentional set were necessary for complex-scene perception. In the multievent conditions, many changes in set are required on each trial, because observers had to moves eye and attention throughout the display. Yet, the cost of perceiving multiple events was not large; it was not a missed gorilla (Simons \& Chabris, 1999). One could say that bottom-up processing driven by the four types of event tokens was somewhat efficient. 


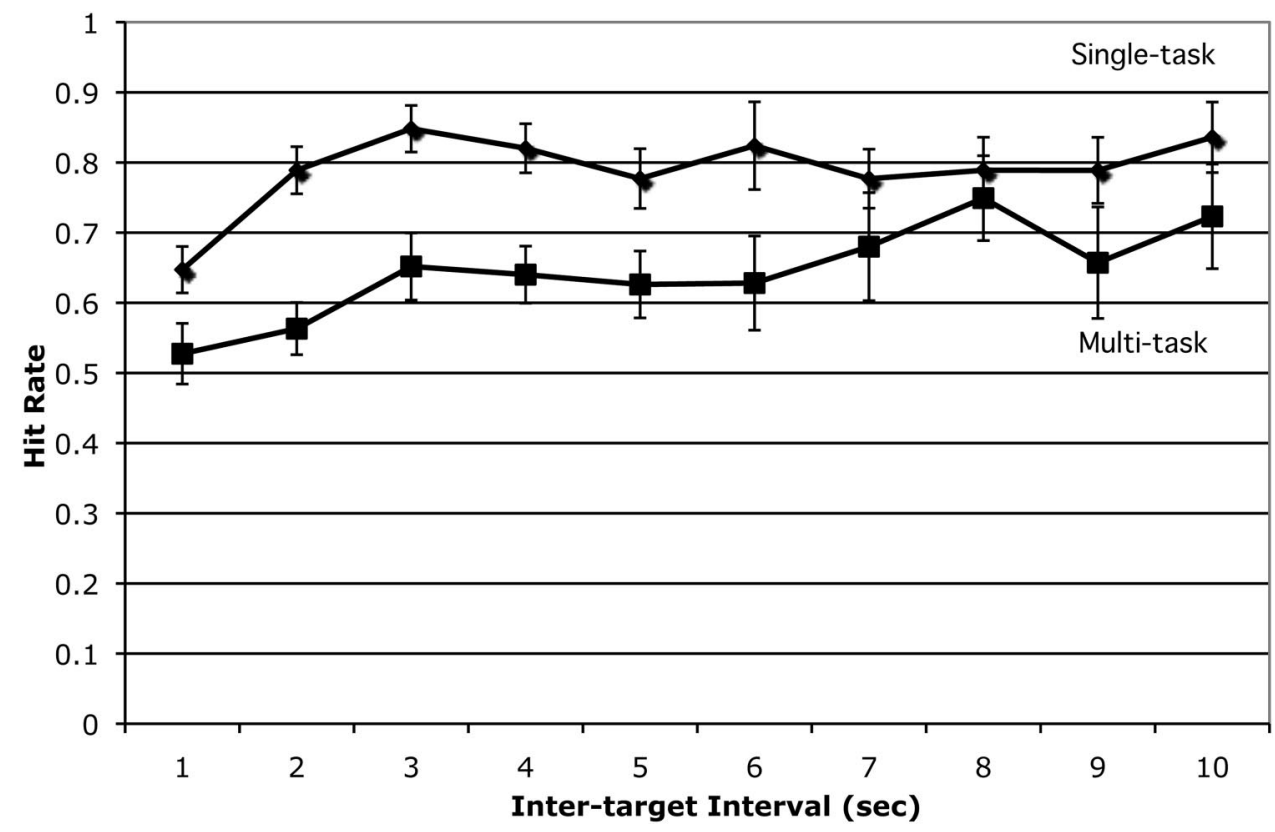

Figure 3. Proportion hits for each ITI window in Experiment 1, with standard errors.

\section{Experiment 2}

Experiment 1 produced the multitasking cost predicted by the attentional-set hypothesis, but our tentative conclusion was that multievent perception was fairly good in the present situation. Observers appear to adopt a reasonably efficient set for multievent perception (MEP). The set may involve strategies for efficient time sharing between temporally overlapping perceptual processes. Such time sharing is likely in natural environments when events have time scales of seconds. The original attentional-set hypothesis was based on the idea that there is a single set. However, the hypothesis may need to be modified to include the idea of an attentional set for MEP that is less efficient but still generally effective.

GIven the fairly efficient MEP observed in Experiment 1, we changed perspectives and considered the basis of MEP. What types of factors might support reasonably efficient MEP in complex scenes? One possibly general and important factor is spatial organization of task. Spatial organization means that events of the same type are grouped or located in the same region. This general factor appears to be essential in many domains, and to be embedded in our cultural thinking about scenes. When spaces are designed, tasks or purposes are assigned to specific regions (e.g., rooms in homes, regions in urban neighborhoods or factories or campsites). For example, urban design devotes separate lanes to vehicles and to pedestrians, and separate spaces for sitting and for playing. Mixing functions across locations could produce perceptual chaos. In scene-perception research, the learned organization of locations is a new and relevant topic (e.g., Torralba, Oliva, Castelhano, \& Henderson, 2006). In the task switching literature, switching costs for simple tasks can be eliminated or reduced by the use of location as a task cue (Arbuthnott \& Woodward, 2002; Mayr \& Bryck, 2007).

Does reasonably efficient MEP depend on the spatial organization of the tasks? We reduced spatial organization in the multitask condition of Experiment 2. In the new, distributed multitasking condition, the tokens for different tasks were distributed throughout the display. Figure 4 shows the old and new multitask displays. Note that spatial organization can be viewed as a bottom-up factor that can influence attentional set. The hypothesis was that spatial organization would be important for MEP, and that multitasking costs would be greater in this experiment than in Experiment 1.

\section{Method}

The method was similar to Experiment 1, with the exception of the distribution of task tokens in the multitasking displays. Whereas each token stream was assigned to its task cell before each trial in Experiment 1, each token stream was randomly assigned to any one of the four cells in Experiment 2, with the same constraints on cell density as previously. For example, as in Figure $4 b$, one stream of shape tokens (e.g., the diamond-like shape in the upper left) was assigned to that cell, and each token in that stream appeared in a random position of the cell, throughout the trial. Displays cells still contained the same number of token streams $(2,3,3$, or 4$)$ as previously. Also, as can be seen in the Figure 4, task reference information (the critical square, and the color border) was provided in each display cell (as in single-task conditions for the two tasks). Fifteen college students (12 women) participated in exchange for course credit. The data for an additional two participants were not analyzed because they did not pass the training criteria.

\section{Results}

The hit rate was high at the end of training, with one-cell displays and a single task (96.0\%). When stimulus complexity was increased during testing to four cells with a single task, performance was reduced to an average of $79.3 \%, F(1,14)=9.40, p<$ .001. When observers had to monitor four events in the new 


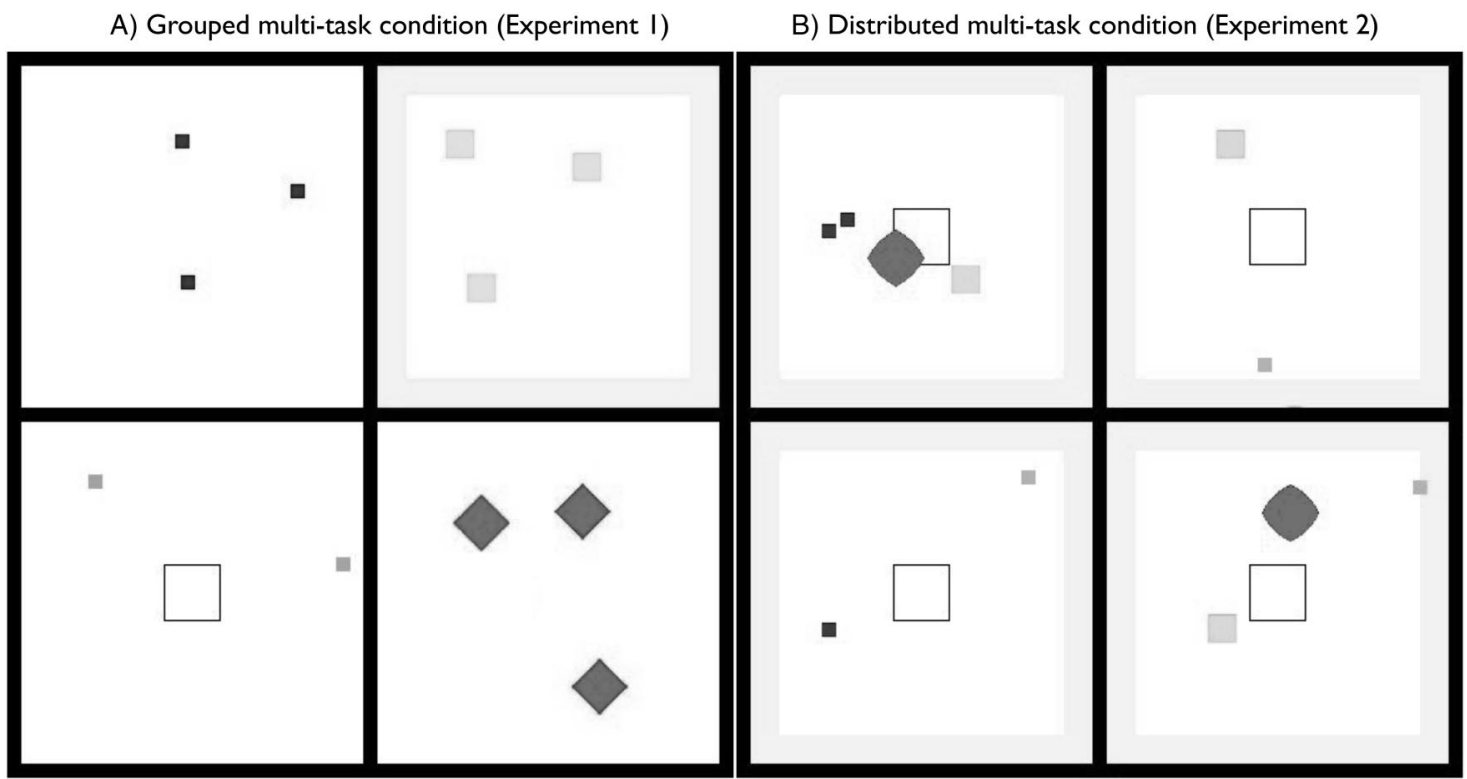

Figure 4. Multi-task conditions from Experiments 1 and 2.

multitasking displays, the hit rate was reduced to $45.2 \%$. This is an absolute cost of $34.1 \%$ : $S E$ of cost $=1.8 \% ; F(1,14)=338.7, p<$ $.001, \eta_{\mathrm{p}}^{2}=.96$. In more concrete terms, subjects went from identifying most targets on single-task trails (6.3 of 8 ) to less than half (3.6 of 8) on multitask trials. This appears to be a fairly profound cost. Compared with Experiment 1, the multitask cost was $20.0 \%$ greater in absolute size, $F(1,28)=31.787, p<.001$, $\eta_{\mathrm{p}}^{2}=.53$. Thus, reducing spatial organization by distributing the tasks across the four cells made the perception of multiple events much more difficult. The results suggest that in complex scene perception, the challenges of switching between events are especially large when events are not spatially organized by type.

Consistency across event type. Table 4 shows hit rates broken down by event type (task). There was the main effect of multitasking reported above, and no main effect of event type $(F<$ $1)$. The interaction of multitasking and event did not approach reliability, $F(3,42)=1.32, p>.20$. Single-task performance was higher than multitask performance for all 4 event types. Note that in this experiment, there were four critical locations for the location event, in both the single-tasking and multitasking conditions (see Figure 3), removing the confound in Experiment 1. This appears to erase most of the interaction with event type found that experiment. Thus, the multitasking cost was strong in the present experiment, across all event types. In fact, for each event type the costs were greater in magnitude than in Experiment 1.

Table 4

Hit Rates for Each Event Type and Task Condition in Experiment 2

\begin{tabular}{lcccc}
\hline & Color & Shape & Motion & Location \\
\hline Single-event & 0.84 & 0.81 & 0.78 & 0.74 \\
Multievent & 0.46 & 0.46 & 0.41 & 0.47 \\
Cost & 0.38 & 0.35 & 0.37 & 0.27 \\
\hline
\end{tabular}

Effects of intertarget interval (ITI). The data were divided into 1-s ITI windows as previously, capturing $89 \%$ of the postinitial target events, and are shown in Figure 5. In this experiment, the ANOVA produced the main effect of task condition reported above. There was no main effect of ITI, $F(9,126)<1$, but there was an interaction of ITI and task condition, $F(9,126)=2.34, p=$ $.02, \eta_{\mathrm{p}}^{2}=.16$. In the single-task conditions, the main effect of ITI approached reliability, $F(9,126)=1.93, p=.05, \eta_{\mathrm{p}}^{2}=.121$. The decrement relative to baseline was $14.1 \%$ at $1 \mathrm{~s}, t(14)=6.20, p<$ .001 , and $11.8 \%$ at $2 \mathrm{~s}, t(14)=2.80, p=.01$. Performance appears to asymptote after that, from 3-10 s (or perhaps 5-10 s).

In contrast, in the multitasking condition, the effect of ITI was not reliable, $F(9,126)=1.41, p>.10$. The ITI function is fairly noisy, and performance tended to be (unreliably) better at the two shortest ITIs than at baseline $(3.0 \%$ and $9.1 \%$ advantages, respectively; $p$ 's $>.10)$. Thus, the ITI function for distributed grouping was different from that for single-tasking, and different from both task conditions in Experiment 1. The difference in the ITI functions for the two task conditions may relate to whether an attentional set can be reestablished after a target is identified. The combined data will provide stronger evidence on this issue.

Learning effects during the session. Performance was generally constant from the first (early) set of single-task blocks to the final set, averaging $80.5 \%$ and $78.0 \%$, respectively, $t(14)=1.33$, $p>.20$. Thus, single-tasking performance was generally constant across the experiment.

\section{Control Study Examining Perceptibility of Tokens}

We would like to interpret the fairly profound costs for distributed multitasking as a cost of task switching. However, an alternative explanation stems from possible differences in the perceptibility of tokens per se, in the distributed displays. Performance could be low in the new distributed condition because of the tokens are difficult to see, rather than because of task switching. Perhaps 


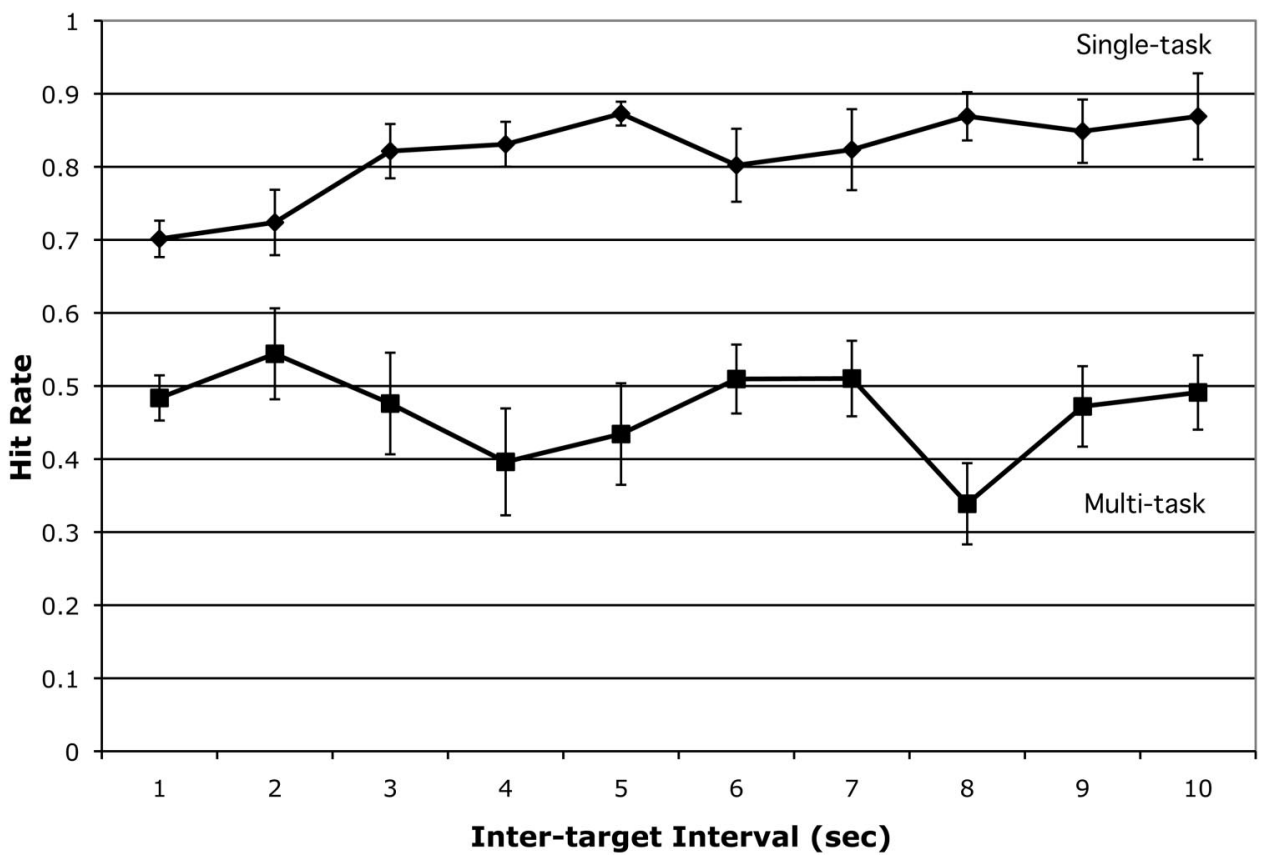

Figure 5. Proportion hits for each ITI window in Experiment 2, with standard errors.

the mixture of different token types within a cell creates a busier display that reduces greatly perceptibility of the tokens themselves.

To test this explanation, we designed a control study to check the perceptibility of tokens. To separate token perceptibility from MEP, we used selective attention instructions with the distributed multitasking displays. The design was the similar to Experiment 2, aside from the multitask displays and instructions. During training with individual tasks, 14 of 16 new observers passed the criteria. The single-tasking condition was the same as previously (including instructions), and the average performance level was $73.3 \%$. In the new condition, the distributed displays (Figure 4B) were used, with two modifications. First, the task was a selective attention task; observers were instructed to detect targets of only one type at a time. There were four separate blocks, one for each event type. Second, to equate target frequencies with the main experiment, there were eight targets of each task type instead of two, and six fewer distractor tokens of each type. This made the overall task (detect 8 tokens) the same as previously. The eight targets could appear in any of the four display cells, so observers had to monitor the entire display.

If the tokens themselves were difficult to perceive, because of the busy nature of the multitasking displays, then the tokens should also be difficult to detect in this selective attention condition. The overall hit rate in this selective attention condition was $88.3 \%$, and performance was high with each task (color: $92.8 \%$, shape: $91.2 \%$, motion: $82.6 \%$, location: $86.7 \%$ ). FA rates were less than $1 \%$. Thus, the targets were not difficult to see in the distributed multitasking displays. In fact, performance was better than each of the single-task conditions in this experiment (which averaged $73.3 \%$, as noted), and better than the single task conditions of the main experiment (see Table 4). The high performance levels in this selective attention condition may occur because only the taskrelevant tokens needed to be processed under the selective atten- tion instruction. If observers can effectively selectively attend to one event type throughout the multitask display, the effective number of distractor tokens is reduced to 28. Most importantly, the results imply that the tokens and the targets of each event type are perceptible.

This control study was not designed to provide a comprehensive assessment of token perceptibility across different conditions. Instead, it was designed to check whether the tokens were perceptible in the distributed multitasking displays. The high level of performance indicates that they were. The large decrements when observers multitasked in the main experiment cannot be explained by difficulties perceiving tokens.

\section{Discussion}

Experiment 2 produced large multitasking costs that were consistent with the idea that spatial organization of task is critical for reasonably efficient MEP. When tasks were no longer organized by cell in the multitasking conditions, performance was considerably lower than previously, falling to a hit rate of less than $46 \%$. Further, there was no evidence of recovery at short ITIs in the distributed multitask condition after a target had been identified. From an attentional-set perspective, this suggests that an efficient set could not be reinstated after target detection.

Overall, Experiments 1 and 2 suggest that there are three levels of efficiency in the single-tasking and multitasking conditions. Optimal efficiency is obtained when there is a single task and observers can use a single attentional set. Efficiency is reduced when observers must change set continuously to handle 4 event types (Experiments 1 and 2). However, if the attentional set involves dedicated regions for each event type (Experiment 1), a reasonably efficient set for MEP can be maintained. When event types are distributed throughout space (Experiment 2), levels of 
perceptual efficiency are markedly lower, and a reasonably efficient set for MEP cannot be established. Interestingly, spatial organization may be a primarily bottom-up factor because it is an aspect of stimulus organization. This factor interacts with the observer's intention to successfully multitask.

\section{Experiment 3}

We interpreted the large multitasking decrement in Experiment 2 in terms of the importance of spatial organization of the visual tasks for MEP. However, the response was also spatial in naturetarget display cell was mapped onto a spatially corresponding key. Does the nature of the response contribute to the spatial organization effect? Could response complexity influence control processes in the present situation? To begin examining these questions, we used a simple, nonspatial response in Experiment 3-observers pressed the spacebar whenever a target occurred, in any cell. Also, to more directly compare two multitasking conditions, we included both the distributed and the grouped multitasking displays.

\section{Method}

There were 3 main task conditions arranged in blocks: Single tasking, grouped multitasking, and distributed multitasking. Each session began with training for each task with one display cell, followed by the four cell test conditions: single-tasking, and then the two multitasking conditions (in order counterbalanced across observers), and then the single-tasking condition again. Training involved, for each task, three trials with feedback and three trials without feedback. The first single-task condition involved one practice trial and one test trial for each event type. Each multitask condition involved four practice trials followed by 12 test trials. The final single-task condition involved two test trials for each event type. The observers were instructed to press the spacebar once whenever they detected a target. A response was scored as a hit if it was the first response to occur within the temporal response window; location could not be scored. Data from 22 observers (11 in each counterbalance group; 14 women in total) were analyzed; data for an additional 3 observers were not included because they failed to meet training criteria.

\section{Results}

The hit rate was high at the end of training with one-cell displays and a single task (96.6\%). When stimulus complexity was increased to 4 cells but a single task, performance was reduced to an average of $85.5 \%, F(1,21)=34.26, p<.001$. This is a higher level of single-task performance than in the previous experiments, possibly because of the simplified response method.

When event types changed but in a spatially organized manner (grouped multitasking), performance was decreased $20.6 \%$ relative to the single-task conditions (to $64.3 \%$; $S E$ of cost $=1.8 \% ; F(1$, $21)=127.49, p<.001)$. Performance was reduced $11.7 \%$ more in the distributed multitasking condition relative to grouped multitasking, to a level of $53.2 \%$ ( $S E$ of cost $=1.8 \% ; F(1,21)=41.41$, $p<.001)$. Thus, even with a response rule that has no spatial component, there are multitasking costs, and the costs is especially high when the tasks are distributed throughout the display. In more concrete terms, the number of targets detected out of eight ranged from 6.1 in single-task conditions, to 5.2 in grouped multitask conditions, to 4.2 in distributed multitasking conditions.

Consistency across event type. Table 5 shows hit rates broken down by event type (task). The main effect of task condition was reliable, $F(2,42)=160.09, p<.001, \eta_{\mathrm{p}}^{2}=0.88$, as was the main effect of event type, $F(3,63)=39.91, p<.001, \eta_{\mathrm{p}}^{2}=0.65$. Also reliable was the interaction of task condition and event type, $F(6,126)=10.48, p<.001, \eta_{\mathrm{p}}^{2}=0.33$. Both the main effect of event type and the interaction can be attributed to the motion event, where performance was low in the two multitasking conditions. In this experiment and in the previous experiments, performance for the motion event tended to be lowest of the single-event conditions, and costs tended to be higher. The costs were especially high in the present experiment. However, there were substantial costs for all four event types.

Effects of intertarget interval (ITI). The ITI data for 1-s windows are shown in Figure 6 (capturing 91\% of the postinitial target events). In this experiment, there was a main effect of ITI, $F(9,189)=52.96, p<.001 ; \eta_{\mathrm{p}}^{2}=.72$, and statistically, it was consistent across task condition, $F(18,378)=1.28, p<.10$. The overall decrement relative to baseline was $26.1 \%$ at $1 \mathrm{~s}, t(21)=$ $11.07, p<.001$, and $11.4 \%$ at $2 \mathrm{~s}, t(21)=4.44, p<.001$. Thus, there was a substantial decrement when targets were close together in time in this experiment. Further analyses were conducted with order-group as a factor (observers receiving grouped multitasking first vs. those receiving distributed multitasking first). However, none of the interactions involving this variable approached reliability.

Although the interaction of task and ITI was not reliable, there is some evidence of differences between ITI functions, and in particular, a somewhat slower recovery in the distributed multitasking condition-reduced performance at the 3-s ITI. To provide a more powerful look at the ITI functions, we regrouped and combined the ITI data across experiments, and present them in the next major section.

Learning effects during the session. Again, performance was similar from the first set of single-task blocks to the final set, averaging $80.5 \%$ and $78.0 \%$, respectively, $(t<1)$. Thus, performance was generally constant across the experiment.

\section{Discussion}

Experiment 3 indicates that perceiving multiple events is very difficult when the events were intermixed spatially, even with a simple response rule. Multiple events are easier to perceive when they are spatially organized by task, regardless of the response organization. However, there was also a decrement for grouped multitasking in the experiment; optimal perceptual efficiency was obtained only in single-task conditions.

Table 5

Hit Rates for Each Task and Condition in Experiment 3

\begin{tabular}{lcccc}
\hline & Color & Shape & Motion & Location \\
\hline Single-event & 0.88 & 0.85 & 0.83 & 0.86 \\
Multievent Grouped & 0.72 & 0.75 & 0.41 & 0.71 \\
Multievent distributed & 0.62 & 0.58 & 0.35 & 0.57 \\
Costs (S-G) & 0.15 & 0.10 & 0.42 & 0.15 \\
Costs (G-D) & 0.10 & 0.17 & 0.06 & 0.13 \\
\hline
\end{tabular}




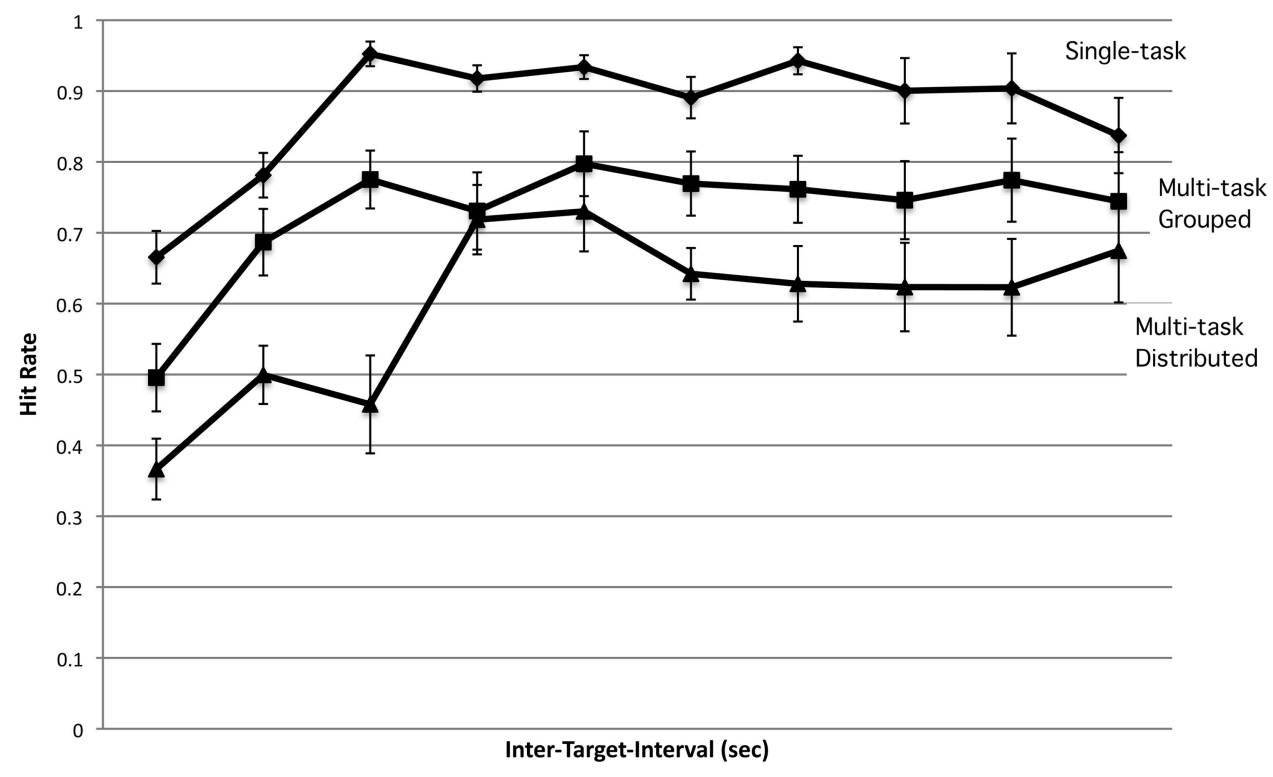

Figure 6. Proportion hits for each ITI window in Experiment 3, with standard errors.

The change to a simple one-button response rule did not alter the overall pattern of results. However, note that overall performance levels increased relative to the previous experiments. The effects of response complexity can be examined further by comparing results between experiments. The combined results are interesting, and include interactions with response complexity, and conditions under which attentional set was optimized.

\section{ITI and Response Complexity Across Experiments}

To further examine the ITI functions and response complexity, we regrouped the data from the three experiments into two mixeddesign "experiments." Each regrouped experiment has two levels of task condition, crossed with two levels of response complexity, and the ITIs $(2 \times 2 \times 10)$. The first regrouped experiment compares single-tasking to grouped multitasking and 4-button responses to 1-button responses, using data from Experiments 1 and 3. The full design and conditions are specified below. ${ }^{4}$ Together, the two regrouped experiments use all of the data from the three experiments.

The figures were formatted to emphasize the systematic functions. Figure 7 shows the data for singe-task and grouped multitask conditions. The two functions are similar in shape, and indicate that the main difference between single-task and grouped multitask conditions is a constant (intercept) effect. For each task condition, there is a linear rise to an asymptotic performance level at $3 \mathrm{sec}$, followed by generally constant performance at $3 \mathrm{~s}$ and beyond. A simple interpretation of the functions is as follows. As a target is detected, detection of temporally close targets in the $1 \mathrm{~s}$ window suffers considerably. Then there is a recovery process causing a linear increase in performance and taking a total of $3 \mathrm{~s}$. Throughout longer ITIs, performance remains high, defining an asymptote. The constant difference between the two task conditions has been explained by differences in the efficiency of single- and multievent perception. The rate of recovery appears similar in the two task conditions; the single- and multievent slopes (increase from
$1-3$ s) were $12.5 \%$ and $10.7 \%$ in the single-task and multitask conditions, respectively ( $F<1$ in a fairly sensitive withinobserver comparison; $S E_{\text {difference }}=2.1 \%$ ).

In further interpretations of the ITI functions, the asymptotic levels at 3-10 s provide a good landmark - that is, they indicate the optimal level for the task condition, where set has been optimized. These levels appear as dashed lines in the figure. Relative to this landmark, the decrements in performance at short ITIs can be interpreted as a temporary loss of optimal efficiency, or perhaps loss of set. Further, the recovery slopes can be reframed, in terms of extents of recovery. In Figure 7, the extents of recovery were $20.2 \%$ and $20.4 \%$, for single-task and multitask, respectively. These extents can be compared with other conditions.

The similarity of the function shapes for single-tasking and grouped multitasking, and in particular the similar extent of recovery, is potentially interesting because these conditions differ in the requirements for attentional set. After target detection in single-task conditions, observers need only prepare for (or reprepare for) one event type, and the next target will be of that same event type. In contrast, in the grouped multitask condition, observers must eventually prepare for four events types, and the next target is most often a change in event type. The similar recoveries in these conditions is significant and considered further in the General Discussion.

Response complexity modifies the ITI effects. There was a substantial difference between the more complex (four-button; Experiment 1) and simpler conditions (one-button; Experiment 3), as can be seen in Figure 8. These data are separated by response complexity and combined across the two task conditions. The difference is the slopes and the extents of the recovery process-a faster and greater recovery with the simpler 1-button response of

\footnotetext{
${ }^{4}$ Task Condition (single task versus grouped multitask) $\times$ Response (four buttons versus one), with the following conditions: Single task and grouped multitask from Experiments 1 and 3.
} 


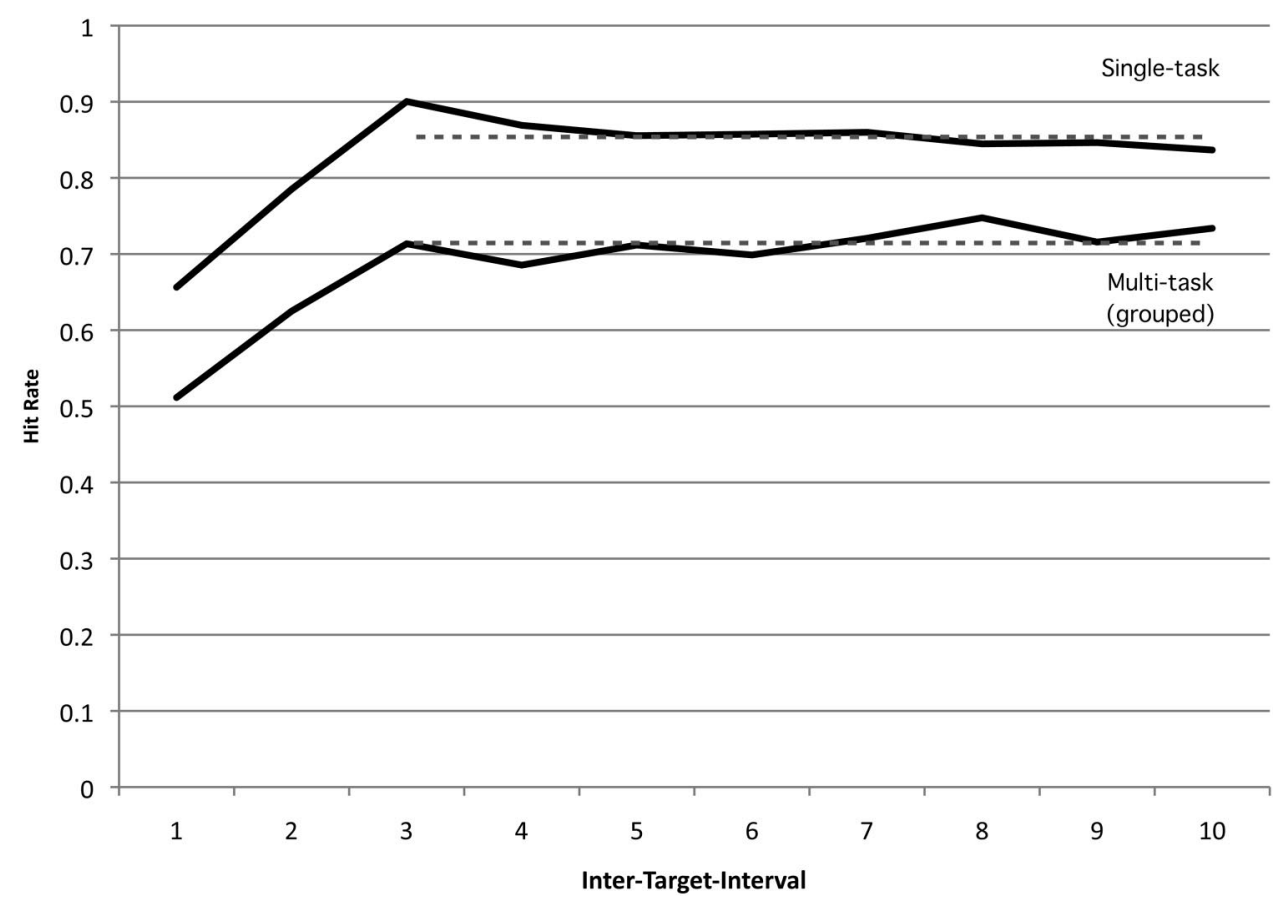

Figure 7. Means of single-task and grouped multitask conditions of Experiments 1 and 3. (Error bars have been removed to emphasize the functions; variability is represented in the previous figures.)

Experiment 3 (14.2\% slope, $25.6 \%$ extent) than with the 4-button response of Experiment 1 (8.1\% per s slope, $15.1 \%$ recovery; $p<$ .01 ). There is less (and slower) recovery when observers must reinstate the complex screen-to-response mapping required with the spatially organized, four-button response rule. Overall perceptual efficiency is higher with the simpler response, perhaps because the easier response rule allows a more effective perceptual set to be maintained.

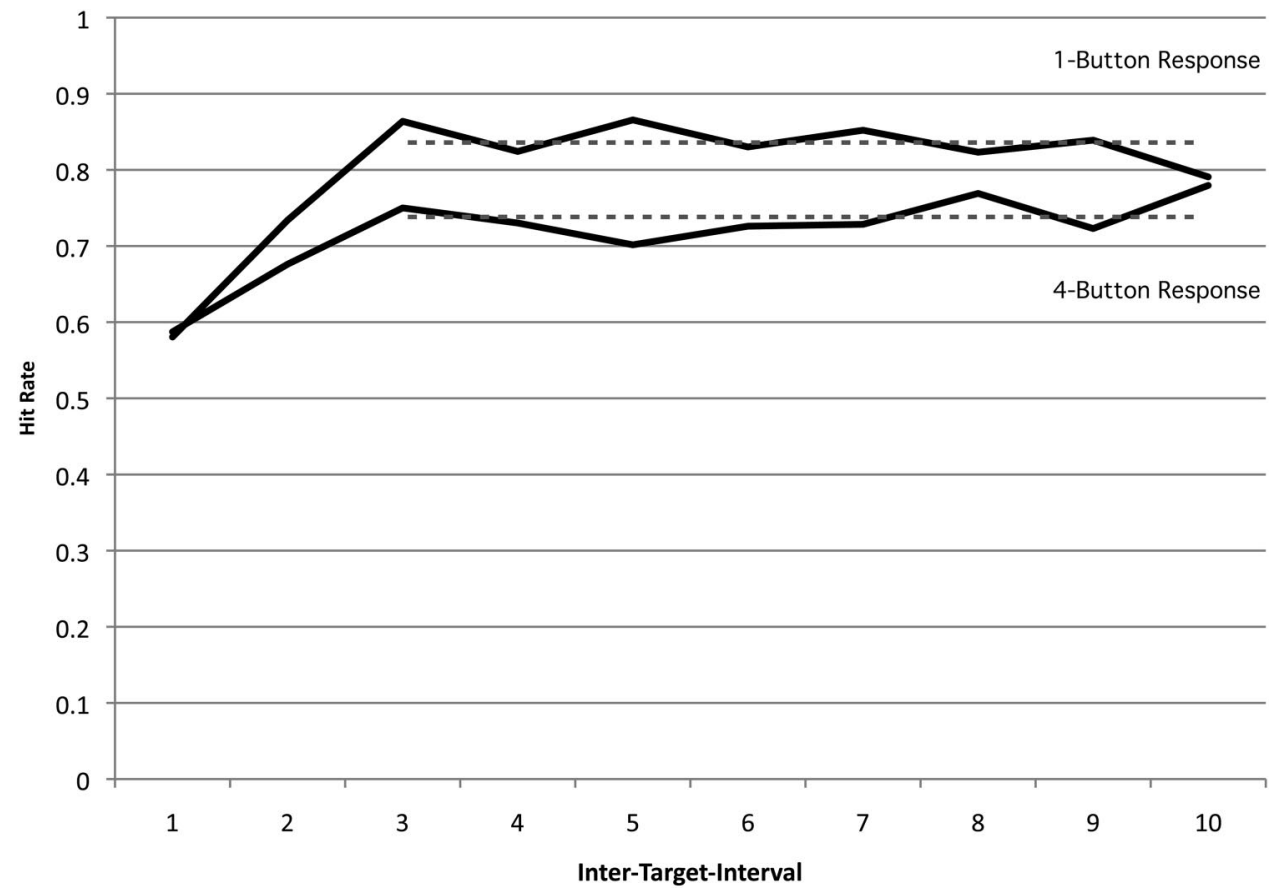

Figure 8. Mean of high response complexity (Experiment 1 ) and low complexity (Experiment 3 ) conditions; single-task and grouped multitask data only. 
The overall effects of task condition and response complexity shown above capture most of the variation in ITI data for these conditions. The significant task effects were reported with the original experiments. Further effects were evaluated in a mixeddesign ANOVA. The effect of response complexity was reliable, $F(1,35)=6.23, p=.02$. On the other hand, none of the overall interactions involving ITI approached significance. ${ }^{5}$

We now turn to the second regrouped experiment, which compares single-task and distributed multitask conditions (Experiments 2 and 3). ${ }^{6}$ The data for the two task conditions are shown in Figure 9. The single-task function is similar to previously, showing a 3-s recovery to asymptote followed by a generally constant asymptotic period. The extent of recovery is $19.3 \%$. In contrast, performance is much lower overall for the distributed multitask condition, and recovery is less definitive. Recovery may take as long as $5 \mathrm{~s}$, and the amount recovered is smaller (12.0\% extent). The slope for the first three ITIs was higher for the single-task condition $(11.0 \%$ per $\mathrm{s})$ than for the distributed multitask condition (2.6\% per $\mathrm{s}, p=.01)$.

This same data-set can be analyzed for response complexity effects. As can be seen in Figure 10, there are marked differences in recovery slopes and extents. With one button responses, recovery is robust for the first $3 \mathrm{~s}$ (9.5\% slope, $25.8 \%$ extent), and appears to continue to $5 \mathrm{~s}$. With four buttons, however, little if any recovery occurs (slope $=2.8 \%$, extent $=5.5 \%$ ). The differences in recovery are marked in the 4-button distributed multitasking condition, as will now be seen.

Task condition and response complexity interact in this set of data. In the ANOVA, there were effects of task (reported in the original experiments) and response complexity, $F(1,35)=$ 11.33, $p=.002$. In addition, there was an interaction of task, response complexity, and ITI, $F(9,306)=2.62, p<.001, \eta_{\mathrm{p}}^{2}$ $=0.07$. Therefore, we also present the 4 ITI functions defined by task condition and response. Because the individual functions are somewhat noisy (and were presented, in the slightly different groupings of Experiments 2 and 3), we grouped adjacent ITI windows to provide the more systematic functions shown in Figure 11.

The single-task functions reiterate that there is an initial recovery phase with a larger extent for the simpler one-button responses than for four buttons. The distributed multitasking functions provide new information, however. First, in the 1-button condition there is a slower but sizable recovery. The extent of the recovery by $3 \mathrm{~s}$ is $27.1 \%$ (calculated as above, from $1 \mathrm{~s}$ windows). In this condition, a set for MEP appears to be constructed, but more slowly than in other conditions. The recovery appears to continue out to $6 \mathrm{~s}$. However, the asymptotic level is still well below single-task levels, and below the grouped multitask conditions (comparable grouped functions are in Figures 6 and 7). In contrast, in the four-button distributed condition there is no evidence of recovery; the recovered extent is actually negative! The combined demands of complex responses and distributed multitask displays may overwhelm control processes, with the result that a MEP set cannot be set in this condition. Consequently, performance remains low throughout the ITIs. We consider interpretations of the ITI functions further below.

\section{General Discussion}

The experiments tested the idea that attentional set is critical in complex scene perception. We manipulated the number of event types and measured overall perceptual efficiency. Consistent with the attentional-set hypothesis, perceptual efficiency was optimal when observers were set for a single event type-hit rates in single-task conditions averaged $81 \%$ across experiments, and asymptotic performance at moderate and longer ITIs reached $91 \%$ (see Figure 11). When observers had to change set continuously to handle four event types in organized locations, performance was reduced by a substantial amount-overall hit rates fell an average of $17 \%$ relative to single task, and asymptotic levels reached only $76 \%$ (see Figure 6). This level of perceptual efficiency is reasonably high, however, and one could say that observers were set to handle four event types fairly well-a set for multiple-event perception (MEP). In fact, an attentional set for multiple grouped events was reinstated as quickly as in single-event conditions (e.g., Figure 7). Only when the spatial organization of the tasks was disrupted by distributing event types did performance fall drastically, bringing hit rates down to $48 \%$ in distributed multitasking conditions (Experiments 2 and 3). This is a miss rate above 50\% and a repeated form of inattentional blindness, in which observers fail to detect targets present in their field of view (cf. Mack \& Rock, 1998; Simons \& Chabris, 1999). Furthermore, the ITI data indicate that there are difficulties establishing a set for MEP in distributed conditions. Set was reinstated slowly but somewhat effectively with simple responses, reaching an asymptote of $64 \%$ in Experiment 3 (see Figure 11). More significantly, MEP could not be optimized with the more complex stimulus-to-response mapping; there was no rise to asymptote, with performance during that period averaging only $45 \%$ (Experiment 2, and Figure 11).

Overall, the range in optimal performance levels indicates that attentional and response set are important factors in scene perception. The results suggest that everyday scene perception can be severely limited in its efficiency, even when all target events are expected and predictable. Although we cannot generalize accurately to all types of complex scenes, the results suggest that efficiency limitations are pervasive in the perception of complex dynamic scenes.

\section{Attentional-Set Approach to Scene Perception}

The core idea of the attentional-set approach is that perception is an active process that depends on the state of the observer. Although early visual processing may be fairly automatic, the later stages of visual and cognitive processing are argued to depend on attentional set. The present results underscore the importance for overall perceptual efficiency of visual processing components related to specific target events. The

\footnotetext{
${ }^{5}$ We also note the small slope differences apparent in the asymptotic periods $(3-10 \mathrm{sec})$ in both figures. Performance in simpler conditions (single task and 1-button response) tends to decrease slightly across the targetless periods, whereas it tends to increase slightly in more complex conditions (multitask and 4-button response). Perhaps these effects reflect slight changes in alertness or set efficiency over time. Similar small effects are seen in the next set of data.

${ }^{6}$ The design is Task Condition (single task versus distributed multitask) $\times$ Response (four buttons versus one), with the following conditions: Single task and distributed multitask from Experiments 2 and 3.
} 


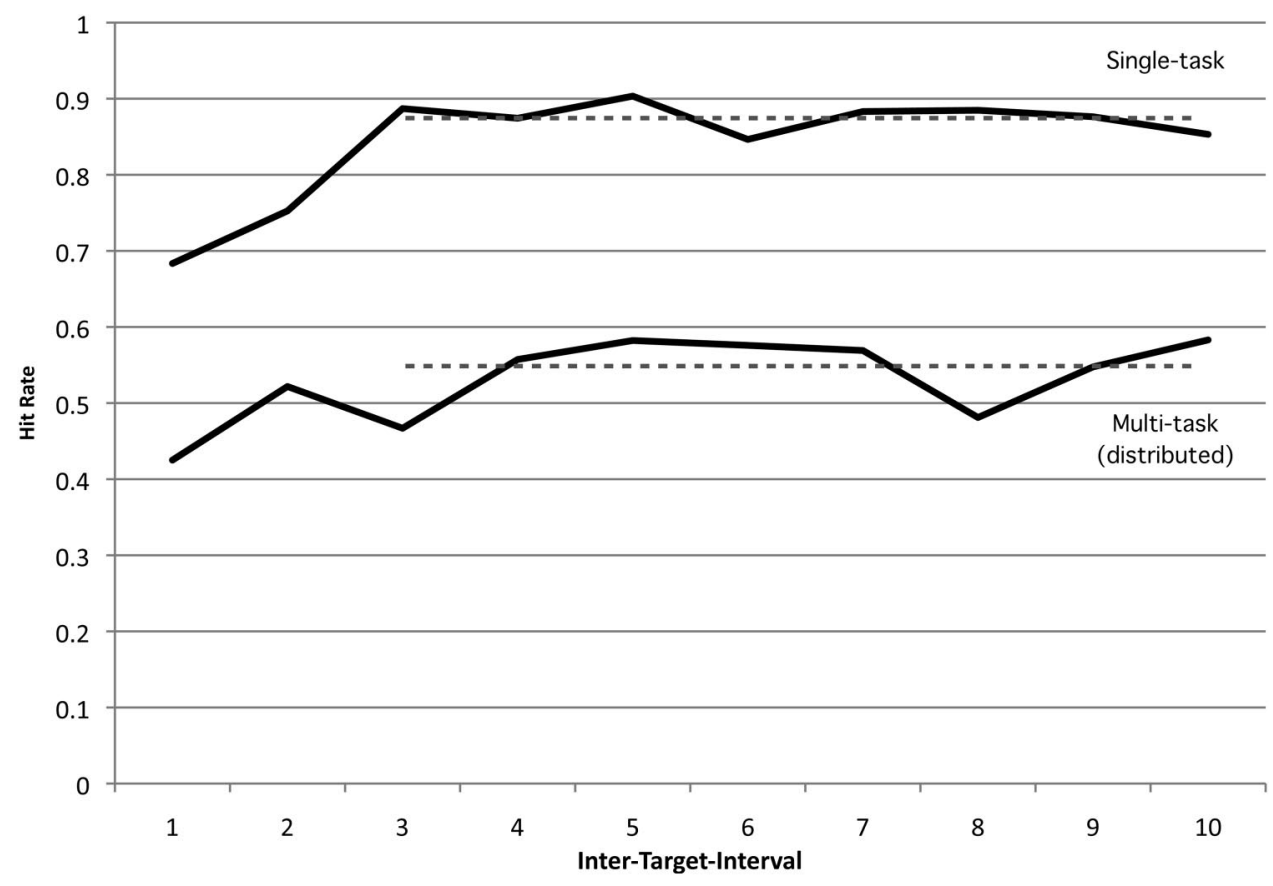

Figure 9. Means of single-task and distributed multitask conditions of Experiments 2 and 3.

experiments also revealed the importance of executive processes related to the response; response complexity determined whether recovery would occur after target detection and the optimal level of performance reached.
The simplest set hypothesis is that performance is optimal with a single attentional set, and this was confirmed in the present experiments. However, we argued that efficiency was reasonably high when there were multiple types of perceptual

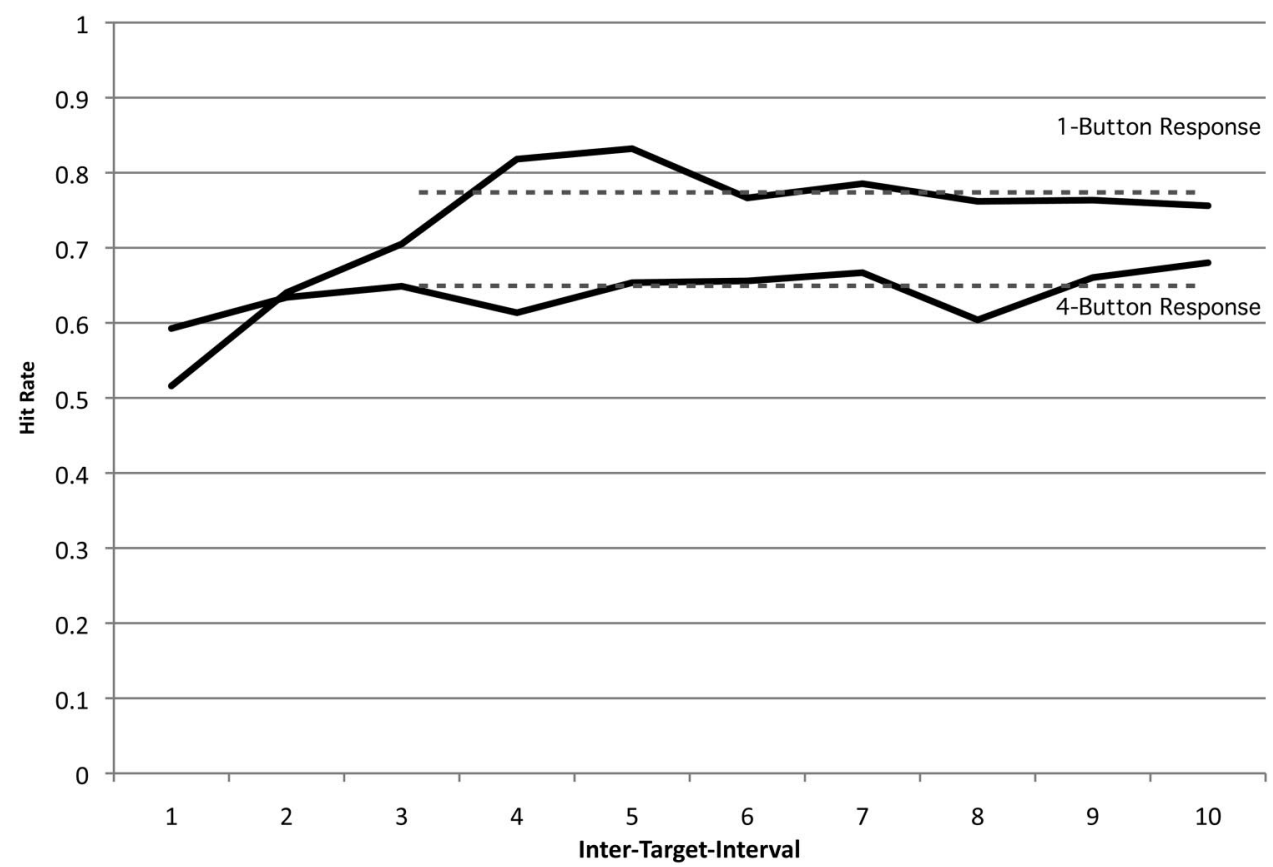

Figure 10. Means of high response complexity (Experiment 2) and low complexity (Experiment 3 ) conditions; single-task and distributed multitask data only. 


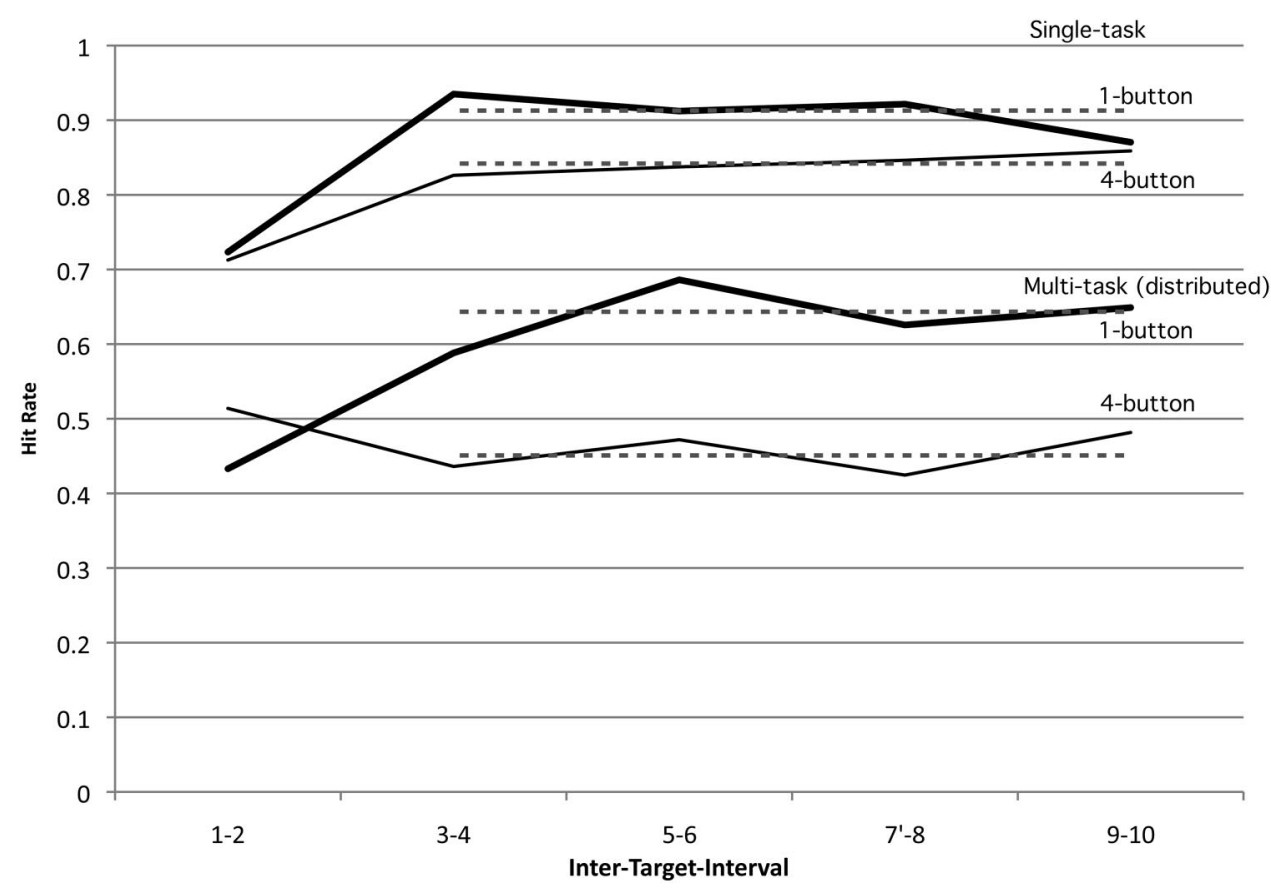

Figure 11. ITI functions for each condition of the regrouped $2 \times 2$, single-task (top two functions) and distributed multitask data (bottom two) only. Adjacent ITI windows have been combined to reduce error.

events, and we suggested the idea of MEP set. MEP set is likely to be important in a complex world, and it has clear boundary conditions, including grouping of event types by location, and complexity of response.

More detailed hypotheses about attentional set in the present situation are developed below. In general, attentional set is thought to influence the ease with which perceptual processes bring target events into consciousness (e.g., Mack, 2003; Most et al., 2005; Simons \& Chabris, 2004). However, observers are unlikely to be aware of set itself. Observers are likely to be aware of goals, with control settings being implemented as the observer intentionally pursues goals, such as watching for a particular type of object or event. Control settings may also be activated by stimuli that are associated with certain actions or goals. Thus, perception is active in the following sense: Entry into awareness (sensitivity of perception), is influenced by control settings related to the observer's goals and recent experiences.

The critical factor of spatial organization deserves further comment. In many ways, it is a stimulus or bottom-up factor rather than top-down factor, because it concerns the organization of the environment. The effects of this factor emphasize the interactive nature of top-down and bottom-up processing (e.g., McClelland \& Rumelhart, 1981; Most et al., 2005; Neisser, 1976; Palmer, 1975); stimulus organization helped observers more effectively meet their goal of successful multitasking.

\section{Measuring Scene Perception}

The main measure of perceptual efficiency used here was global, summarizing overall accuracy across the $60 \mathrm{~s}$ trials. A global measure is appropriate because everyday scene perception is a resultant outcome of a composition of more elementary pro- cesses. We argue that overall efficiency for natural composites of processes is a criterion that models of everyday scene perception should address. However, it is also important to decompose performance into component subprocesses, in order to understand the underlying processes.

\section{Explanations in Terms of Underlying Processes}

We present a brief eye-movement explanation and a more detailed attentional explanation. Both explanations are designed to address the three main types of results obtained here. The first type is differences in optimal performance levels, which were produced by task condition (Figures 7 and 11) and by response complexity (Figures 8 and 11). The second type of result is the large decrements at short ITIs, and the third result is the differing recovery rates after that. We begin with the short ITI decrements. A basic explanation is that the decrements occur because resources (foveal fixation in the eye movement account) are focused on the initial target; consequently, subsequent targets in the rest of the display do not receive adequate resources. In most conditions, performance returns to asymptotic levels within $3 \mathrm{~s}$, because the allocation of resources to the rest of the display is restored.

\section{Eye-Movement Model}

In this explanation the restoration of resources involves reestablishing a scanning pattern that is optimal for each event type. In single-event conditions there is one scanning pattern that is restored in $3 \mathrm{~s}$; once restored this pattern produces high optimal levels of performance. In grouped multievent conditions, the scanning pattern is restored for the next display cell and task in $3 \mathrm{~s}$. The pattern must change again for each display cell (event type), and 
this could cause the moderate level of asymptotic performance that was observed. In distributed multievent conditions, the event types are not organized by cell, increasing the difficulty of matching scanning pattern to event types. With a simple response (see Figure 11 ), it is possible that spontaneous groupings of event types are created over the slow, 6-s recovery period. Complex responses, on the other hand, may drain executive functions and thus prevent development of spontaneous groupings, so that there is no optimization of scanning.

\section{An Attentional-Set Model}

The attentional explanation follows similar ideas but we add details from models of visual search, because the present task can be viewed as an instance of visual search in general. In models of visual search (e.g., Wolfe, Cave, \& Franzel, 1989; Zelinsky, 2008), the search process can be divided into several subprocesses. The first set of processes initiate the search, as event tokens appear at the beginning of a trial. The tokens should cause descriptions of target-relevant features (target templates) to be activated. However, because targets did not occur during the initial portion of a trial, search initiation would not influence performance in the present experiments. Use of the target templates defines the second phase of processing. The templates would include information about how a target changes over time. And, to allow observers to detect targets at various eccentricities, the templates should exist at multiple levels of scale (Zelinsky, 2008). Examples of possible template properties are given below. ${ }^{7}$ The template is applied to the stimulus-world in a scanning process in which the template is compared with features extracted from the display. When a display item matches the template to a reasonable degree, further processing is triggered, including a shift of additional attention to the item's location. If the match continues to be high, the item will be admitted to consciousness as a likely target. Processing would then continue until a decision about the item is made (target or distractor). At this point, resources have been focused on the target location, with the consequence that other targets occurring soon are more likely to be missed (the decrement at short ISI's). In most cases, the scanning process resumes and becomes optimized within seconds. A critical issue is how templates for differing event types are used in this scanning process.

The success of the scanning process appears to be well indexed by the asymptotic levels for the respective conditions. These levels indicate that even in single-task conditions, observers miss some targets. In single-task conditions, misses could occur because the complete set of tokens cannot be scanned quickly enough, with the result that some target events occur without being matched against the target template. The miss rate increases when multiple target templates must be used in multitask conditions, and when a complex response rule must be maintained. The need to use multiple templates is a primary cause of multitask costs. Consider first the grouped multitask conditions.

Perhaps the simplest assumption about scanning is that one template is used at a time, and the templates are switched as the observer begins to process a new display cell (with a new category of event types). For explaining the grouped multitasking cost, the critical assumption is that during the template switch, no matching for targets would take place. These interruptions in scanning could increase the miss rate. The rapid recovery at early ITIs for grouped multitask conditions could be explained by assuming that observers need to load only one target template, the one appropriate for the next display cell to be scanned. The selection of which template to use is made rapidly, perhaps driven by stimulus information. The ability to quickly switch templates with new display cells may be the basis of the fairly efficient attentional set for MEP in grouped conditions.

An alternative assumption involves more general control settings - either a more general template for multiple event types, or multiple templates that are matched against stimulus items in parallel manner. In this model, the decrement for grouped multitasking could be explained by the additional difficulty of using more general settings. However, this model does not explain the equally fast recovery, after detection of a target, in grouped multitasking and single-task conditions. It seems that one template should be reset faster in single task conditions than multiple templates or more general templates in multitask conditions. Therefore, we favor the idea that a single template is used, and that it is switched rapidly in grouped multitask conditions.

Returning to the single-template scanning model, consider now the distributed multitask condition, in which events are not spatially organized. To handle each event type within a region of the display, the scanning process would have to continually change target templates. The more frequent template changes explains the lower level of performance in the distributed relative to the grouped conditions. The slow but sizable recovery for distributed multitasking when the response is simple (see Figure 11) could occur because observers create spontaneous groupings of similar event types and are able to use these groups to swap target templates in a fairly efficient manner. Note that within a trial, each event-stream (sequence of tokens) was assigned to a particular cell. Observers may learn the distribution on each trial. As the groupings become organized over about $6 \mathrm{~s}$, the MEP set becomes optimized and distributed multitasking approaches the efficiency of grouped multitasking.

With the added complexities of four-button responses, however, the challenge for the processes that organize template switching appears to be too great. Perhaps there is a single control process that coordinates template switching and response interpretation, and this process is overwhelmed. There could be competition between the stimulus organization and response mapping-between the spontaneous, stimulus-driven organization of task groups, and the need to map from display cells to keys.

\section{A Bottom-Up Explanation?}

An alternative to our top-down emphasis is the idea that bottomup, stimulus-driven processing determines performance in the respective conditions. Could the event-switching costs be explained by bottom-up mechanisms? One possible approach is to posit priming processes that alter efficiency between conditions. For example, the consistency of event types in single-task conditions might result in stronger priming of target templates for that event type than in multitask conditions. There are two problems for this account. First, priming does not seem strong enough of a mecha-

\footnotetext{
${ }^{7}$ Possible features for each task: Location task, proximity to goal box; motion task, abruptness of movement (speed/extent of movement); color task, increase of yellowness; shape task, increase in convexity.
} 
nism to produce the large multievent costs; even in multievent conditions, each event type occurs often and therefore there is no reason for large priming differences. Second, priming does not explain the similar rates of ITI recovery in single-event and grouped multievent conditions, because priming should be stronger (causing a quicker return to optimal levels) in single-event conditions than in the multievent conditions.

\section{Is a Four-Cell Display a Scene?}

The present displays are not the same as natural scenes. Is it reasonable to think of the present displays as representative of scene perception? The motivation for using display cells stemmed from our interest in the surveillance process, in which observers scan scenes looking for critical categories of events. In the surveillance profession, the use of multiple cells is a standard practice. Because security is important, the fact that surveillance operators use multiple cells suggests (anecdotally) that it is a reasonable and efficient way of representing information about events and scenes. Furthermore, psychological evidence suggests that multicell displays capture significant aspects of scene perception, including the perception of layout and meaning (e.g., Sanocki, Michelet, Sellers, \& Reynolds, 2006; Potter \& Fox, 2009). Theories of scene perception assume that scene representations are derived from separate "snapshots" (e.g., Hochberg, 1978), and there is integration across cells (Sanocki et al., 2006). These observations support the assumption that the multiple display cell presentation is a useful approach

The present displays lacked background, unlike natural scenes. Background layout introduces additional complexities to scene perception and could compound perceptual difficulties. Therefore, presence of background could increase the importance of attentional set.

\section{Surveillance and the Design of Complex Scene Perception}

The present experiments are representative of the surveillance process and illustrate that scene perception can be severely limited, even with known, predictable target events. The results indicate that several factors limit perceptual efficiency in scene perception and surveillance: The need to monitor multiple event categories, the need to make complex responses, the presence of multiple targets close in time, and the absence of task-specific locations. Further research on these phenomena should serve both psychological theory and human security.

\section{References}

Arbuthnott, K. D., \& Woodward, T. S. (2002). The influence of cue-task association and location on switch cost and alternating-switch cost. Canadian Journal of Experimental Psychology, 56, 18-29.

Baillargeon, R. (2004). Infants' reasoning about hidden objects: Evidence for event-general and event-specific expectations. Developmental Science, 7, 391-414.

Balcetis, E., \& Dunning, D. (2006). See what you want to see: Motivational influences on visual perception. Journal of Personality and Social Psychology, 91, 612-625. doi:10.1037/0022-3514.91.4.612

Broadbent, D. E. (1977). The hidden pre-attentive process. American Psychologist, 32, 109-118.
Bruner, J. S. (1957). On perceptual readiness. Psychological Review, 64 123-152. doi:10.1037/h0043805

Chun, M. M., \& Potter, M. C. (1995). A two-stage model for multiple target detection in rapid serial visual presentation. Journal of Experimental Psychology: Human Perception and Performance, 21, 109-127. doi:10.1037/0096-1523.21.1.109

Folk, C. L., Remington, R. W., \& Wright, J. H. (1994). The structure of attentional control: Contingent attentional capture by apparent motion, abrupt onset, and color. Journal of Experimental Psychology: Human Perception and Performance, 20, 317-329. doi:10.1037/0096-1523.20 .2 .317

Gao, T., Newman, G. E., \& Scholl, B. J. (2009). The psychophysics of chasing: A case study in the perception of animacy. Cognitive Psychology, 59, 154-179. doi:10.1016/j.cogpsych.2009.03.001

Goldstone, R. L., Braithwaite, D. W., \& Byrge, L. A. (2012). Perceptual learning. In N. M. Seel (Ed.), Encyclopedia of the sciences of learning (p. 872). Heidelberg, Germany: Springer Verlag.

Greene, M. R., \& Oliva, A. (2009). Recognition of natural scenes from global properties: Seeing the forest without representing the trees. Cog nitive Psychology, 58, 137-176. doi:10.1016/j.cogpsych.2008.06.001

Henderson, J. M., \& Hollingworth, A. (2003). Eye movements, visual memory, and scene representation. In M. A. Peterson and G. Rhodes (Eds.), Analytic and holistic processes in the perception of faces, objects, and scenes (pp. 356-383). New York, NY: Oxford University Press.

Hespos, S. J., \& Baillargeon, R. (2001). Reasoning about containment events in very young infants. Cognition, 78, 207-245.

Hochberg, J. E. (1978). Perception (2nd ed.). Englewood Cliffs, NJ: Prentice-Hall.

Kiesel, A., Steinhauser, M., Wendt, M., Falkenstein, M., Jost, K., Philipp, A. M., \& Koch, I. (2010). Control and interference in task switching-A review. Psychological Bulletin, 136, 849-874. doi:10.1037/a0019842

Lavie, N., Hirst, A., \& de Fockert, J. W., \& Viding, E. (2004). Load theory of selective attention and cognitive control. Journal of Experimental Psychology: General, 133, 339-354. doi:10.1037/0096-3445.133.3.339

Leber, A. B., Kawahara, J., \& Gabari, Y. (2009). Long-term abstract learning of attentional set. Journal of Experimental Psychology: Human Perception and Performance, 35, 1385-1397. doi:10.1037/a0016470

Macdonald, J. S. P., \& Lavie, N. (2008). Load induced blindness. Journal of Experimental Psychology: Human Perception and Performance, 34 1078-1091. doi:10.1037/0096-1523.34.5.1078

Mack, A. (2003). Inattentional blindness: Looking without seeing. Current Directions in Psychological Science, 12, 180-184.

Mack, A., \& Rock, I. (1998). Inattentional blindness. Cambridge, MA: MIT Press.

Mayr, U., \& Bryck, R. L. (2007). Outsourcing control to the environment: Effects of stimulus/response locations on task selection. Psychological Research, 71, 107-116.

McClelland, J. L., \& Rumelhart, D. E. (1981). An interactive model of context effects in letter perception: Part 1. An account of basic findings. Psychological Review, 80, 375-407.

Michotte, A. (1991). The emotions regarded as functional connections. G., Thinès, A., \& Costall, G., Butterworth (Eds.), Michotte's experimental phenomenology of perception (pp. 103-116). Hillsdale, NJ: Erlbaum. Original published in 1950.

Monsell, S. (2003). Task switching. Trends in Cognitive Sciences, 7, 134-140. doi:10.1016/S1364-6613(03)00028-7

Most, S. B., Scholl, B. J., Clifford, E. R., \& Simons, D. J. (2005). What you see is what you set: Sustained inattentional blindness and the capture of awareness. Psychological Review, 112, 217-242. doi:10.1037/0033295X.112.1.217

Neisser, U. (1967). Cognitive psychology. East Norwalk, CT: AppletonCentury-Crofts.

Neisser, U. (1976). Cognition and reality: Principles and implications of cognitive psychology. San Francisco, CA: W. H. Freeman. 
Palmer, S. E. (1975). The effects of contextual scenes on the identification of objects. Memory \& Cognition, 3, 519-526. doi:10.3758/BF03197524

Postman, L., \& Bruner, J. S. (1949). Multiplicity of set as a determinant of perceptual behavior. Journal of Experimental Psychology, 39, 369-377. doi: $10.1037 / \mathrm{h} 0058224$

Potter, M. C., \& Fox, L. F. (2009). Detecting and remembering simultaneous pictures in rapid serial visual presentation. Journal of Experimental Psychology: Human Perception and Performance, 35, 28-38. doi: 10.1037/a0013624

Raymond, J. E., Shapiro, K. L., \& Arnell, K. M. (1992). Temporary suppression of visual processing in an RSVP task: An attentional blink?. Journal of Experimental Psychology: Human Perception and Performance, 18, 849-860. doi:10.1037/0096-1523.18.3.849

Sanocki, T., Michelet, K., Sellers, E., \& Reynolds, J. (2006). Functional representations of spatial layout can consist of independent pieces. Perception \& Psychophysics, 68, 415-427.

Sanocki, T., \& Oden, G. C. (1991). Adjustments on representations of familiar patterns: Change over time and relational features. Perception \& Psychophysics, 50, 28-44. doi:10.3758/BF03212203

Schneider, W., \& Shiffrin, R. M. (1977). Controlled and automatic human information processing: I. Detection, search, and attention. Psychological Review, 84, 1-66. doi:10.1037/0033-295X.84.1.1

Schyns, P. G., Goldstone, R. L., \& Thibaut J. P. (1998). The development of features in object concepts. Behavioral and Brain Science, 21, 1-17. doi:10.1017/S0140525X98000107

Simons, D. J., \& Chabris, C. F. (1999). Gorillas in our midst: Sustained inattentional blindness for dynamic events. Perception, 28, 1059-1074. doi: $10.1068 / \mathrm{p} 2952$

Sulman, N., Sanocki, T., Goldgof, D., \& Kasturi, R. (2012). Display-based limitations in human video surveillance monitoring. Manuscript under review.

Torralba, A., Oliva, A., Castelhano, M., \& Henderson, J. (2006). Contextual guidance of eye movements and attention in real-world scenes: The role of global features in object search. Psychological Review, 113, 766-786. doi:10.1037/0033-295X.113.4.766

Tsal, Y., \& Benoni, H. (2010). Diluting the burden of load: Perceptual load effects are simply dilution effects. Journal of Experimental Psychology: Human Perception and Performance, 36, 1645-1656. doi:10.1037/ a0018172
Tsotsos, J. K. (1990). Analyzing vision at the complexity level. Behavioral and Brain Sciences, 13, 423-445. doi:10.1017/S0140525X00079577

Tsotsos, J. K. (2001). Complexity, vision and attention. In M. Jenkin \& L. Harris (Eds.), Vision and attention (pp. 105-128). New York, NY: Springer Verlag.

Tversky, B., Zacks, J. M., \& Hard, B. M. (2008). The structure of experience. In T. Shipley \& J. M. Zacks (Eds.), Understanding events (pp. 436-464). Oxford, UK: Oxford University Press.

Vandierendonck, A., Liefooghe, B., \& Verbruggen, F. (2010). Task switching: Interplay of reconfiguration and interference control. Psychological Bulletin, 136, 601-626. doi:10.1037/a0019791

Van Loy, B., Liefooghe, B., \& Vandierendonck, A. (2010). Cognitive control in cued task switching with transition cues: Cue processing, task processing, and cue-task transition congruency. The Quarterly Journal of Experimental Psychology, 63, 1916-1935. doi:10.1080/ 17470211003779160

Walker, S., Stafford, P., \& Davis, G. (2008). Ultra-rapid categorization requires visual attention: Scenes with multiple foreground objects. Journal of Vision, 23, 1-12.

Walther, D. B., \& Li, F. F. (2007). Task-set switching with natural scenes: Measuring the cost of deploying top-down attention. Journal of Vision, 7, 1-12. doi:10.1167/7.11.9

White, R. C., \& Davies, A. A. (2008). Attention set for number: Expectation and perceptual load in inattentional blindness. Journal of Experimental Psychology: Human Perception and Performance, 34, 1092 1107. doi:10.1037/0096-1523.34.5.1092

Wolfe, J. M., Cave, K. R., \& Franzel, S. L. (1989). Guided search: An alternative to the feature integration model for visual search. Journal of Experimental Psychology: Human Perception and Performance, 15, 419-433. doi:10.1037/0096-1523.15.3.419

Zacks, J. M., \& Tversky, B. (2001). Event structure in perception and conception. Psychological Bulletin, 127, 3-21. doi:10.1037/0033-2909 .127 .1 .3

Zelinsky, G. J. (2008). A theory of eye movements during target acquisition. Psychological Review, 115, 787-835. doi:10.1037/a0013118

Received August 15, 2011

Revision received April 27, 2012

Accepted May 11, 2012 